\title{
Liquid-liquid phase separated microdomains of an amphiphilic graft copolymer in a surfactant-rich medium
}

\author{
Xavier Castellvi Corrons ${ }^{1}$, Jeremie Gummel ${ }^{2}$, Johan Smets ${ }^{2}$, Debora Berti ${ }^{1}$ \\ ${ }^{1}$ Department of Chemistry "Ugo Schiff" University of Florence, CSGI, Via della Lastruccia 3, 50019 \\ Sesto Fiorentino Florence, Italy \\ ${ }^{2}$ Strategic innovation and technology, Procter \& Gamble Brussels Innovation Center, Temselaan 100, \\ 1853 Grimbergen, Belgium
}

\section{GRAPHICAL ABSTRACT}

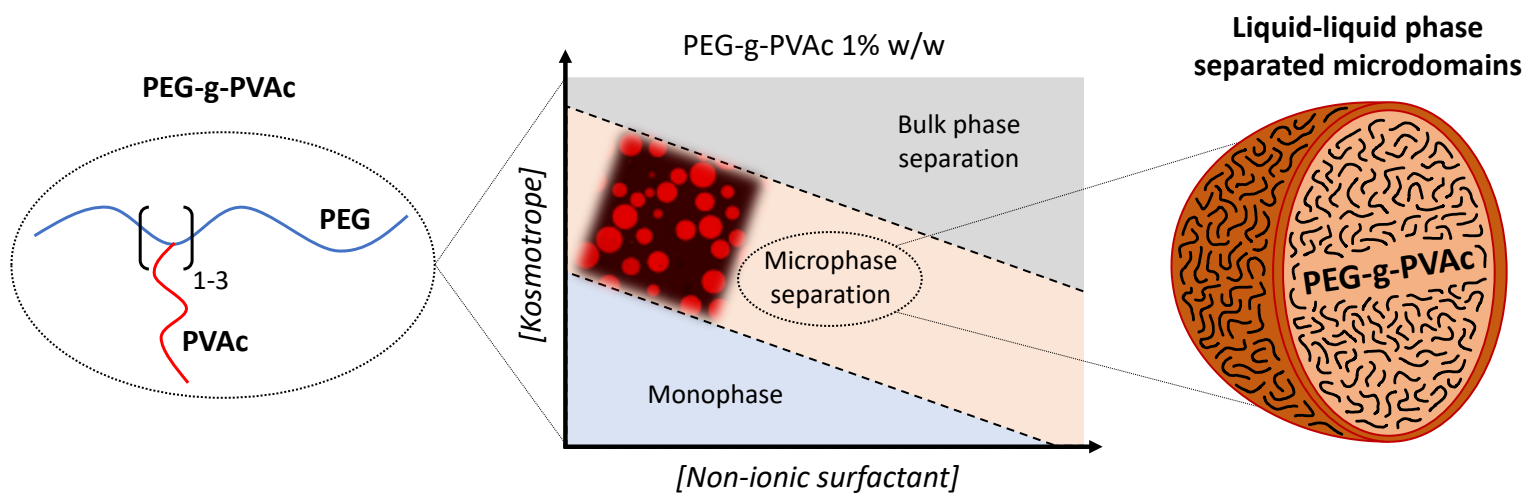

\begin{abstract}
The liquid-liquid phase separation (LLPS) of amphiphilic thermoresponsive copolymers can lead to the formation of micron-sized domains, known as simple coacervates. Due to their potential to confine active principles, these copolymer-rich droplets have gained interest as encapsulating agents. Understanding and controlling the conditions inducing this LLPS is therefore essential for applicative purposes and requires thorough fundamental studies on self-coacervation.

In this work, we investigate the LLPS of a comb-like graft copolymer (PEG-g-PVAc) consisting of a poly(ethylene glycol) backbone ( $6 \mathrm{kDa}$ ) with $\sim 2-3$ grafted poly(vinyl acetate) chains, and a PEG/PVAc weight ratio of 40/60. Specifically, we report the effect of various water-soluble additives on its phase separation behavior. Kosmotropes and non-ionic surfactants were found to decrease the phase separation temperature of the copolymer, while chaotropes and, above all, ionic surfactants increased it. We then focus on the phase behavior of PEG-g-PVAc in the presence of sodium citrate and a $\mathrm{C}_{14-15}$ $\mathrm{E}_{7}$ non-ionic surfactant (N45-7), defining the compositional range for the generation of LLPS microdomains at room temperature and monitoring their formation with fluorescence confocal microscopy. Finally, we determine the composition of the microdomains through confocal Raman microscopy, demonstrating the presence of PEG-g-PVAc, N45-7, and water.

These results expand our knowledge on polymeric self-coacervation, clarifying the optimal conditions and composition needed to obtain LLPS microdomains with encapsulation potential at room temperature in surfactant-rich formulations.
\end{abstract}

KEYWORDS: amphiphilic graft copolymer, liquid-liquid phase separation, critical temperature, coacervate, soft microcapsules 


\section{INTRODUCTION}

Responsive polymers, which reversibly change their properties in response to external stimuli or changes in the medium they are dissolved in [1-4], are increasingly used in several applicative areas, including drug delivery, sensors, and artificial actuators [5,6]. Thermosensitive polymers are probably the most explored class of smart polymers [7,8]; their thermally induced liquid-liquid phase separation depends on the polymer structure as well as on the medium composition, so that both the polymer and the medium can be designed to tune the transition temperature [9-11]. Thermoresponsive polymers that phase separate below a critical temperature are classified as UCST-type (Upper Critical Solution Temperature); conversely, if phase separation occurs above a critical temperature they are classified as LCST-type (Lower Critical Solution Temperature) [12-14]. LCST polymers can be soluble in aqueous media at room temperature, but their solubility decreases upon heating and eventually phase separation into two liquid phases occurs [15]. This entropy-driven phase separation is marked by a cloudy appearance of the sample and is commonly termed as the Cloud Point Temperature (CPT) [16].

Poly(ethyleneglycol) (PEG) is one of the most studied LCST-type homopolymers. Due to a thermallyactivated conformational change of the oxyethylene groups [17], it becomes insoluble above the CPT, which depends on molecular weight $[18,19]$. Many classical studies have addressed the effect of additives in the aqueous medium on its CPT $[20,21]$. Moreover, PEG is commonly used in biomedical applications due to its biocompatibility, stealth effect, and steric stabilization properties [22]. The phase behavior of copolymers containing PEG blocks has also been extensively explored to understand self-assembly and sol-gel transition properties. In previous studies, PEG was conjugated with poly(propyleneoxide) (PPO) to generate the Pluronic or poloxamer family [23-25], but also with poly(N-isopropylacrylamide) (PNIPAM) [26-28], polycaprolactone (PCL) [29-31], poly(Nvinylcaprolactam) (PVCL) [32,33], or poly(lactic-co-glycolic acid) (PLGA) [34,35], to name a few.

More recently, some reports have addressed the phase behavior of grafted poly(ethyleneglycol)-gpoly(vinylacetate) copolymers (PEG-g-PVAc) [36-38]. Graft copolymers, consisting of a backbone and side chains, present interesting properties in solution that can differ from linear diblock copolymers with the same composition $[39,40]$. They have been found to form a variety of self-assembled aggregates in water and organic solvents, which depend on the hydrophilic/hydrophobic features of the backbone and side chains [41-45]. Specifically, PEG-g-PVAc's self-assembly properties depend on the balance between the hydrophobic PVAc grafts and the hydrophilic PEG backbone, as well as in the number and length of the PVAc grafts.

In this contribution, we focus on a PEG-g-PVAc ( $\mathrm{Mw}=18100 \mathrm{Da}$ ) consisting of a comb-like macromolecular structure with a 6000 Da PEG backbone and a grafting point on average every $~ 50$ units of ethylene glycol ( 2-3 grafted PVAc chains per backbone). On the industrial side, this class of copolymers with a low degree of grafting has attracted interest as detergent additives for the formulation of home and beauty-care products. Due to their adsorption properties, they promote a reduction of soil redeposition on garments, especially in hard water conditions [46-48]. The selfassembly properties of a similar PEG-g-PVAc (Mw = $13100 \mathrm{Da}$, 1-2 grafting points every 100 units of ethylene glycol) in water have been reported in the recent past, showing the formation of single-chain nanoparticles (SCNPs) at concentrations below $10 \% \mathrm{w} / \mathrm{w}$ [36], and a progressive structuration of the SCNPs into hierarchically complex systems when increasing the concentration up to $90 \% \mathrm{w} / \mathrm{w}$ [37]. The LCST profile of this PEG-g-PVAc showed a critical temperature of $58{ }^{\circ} \mathrm{C}$ for a concentration of $1 \%$ $w / w$. The application of PEG-g-PVAc self-assemblies for the encapsulation of hydrophobic substances, and specifically fragrances, was also demonstrated $[49,50]$, showing that the molecular properties of 
the guest molecule affect the structure of the copolymer assemblies and their phase behavior in ternary (i.e. PEG-g-PVAc/fragrance/water) systems. The interaction between a PEG-g-PVAc with a different grafting density ( $\mathrm{Mn}=15000 \mathrm{Da}$, one grafting point every $\sim 20$ units of ethylene glycol) and sodium dodecyl sulfate was recently reported too [51], highlighting the synergistic or competitive behavior at the air-water interface, depending on the concentration of anionic surfactant.

PEG-g-PVAc's self-assembly was also investigated in formulations containing surfactant mixtures [38], identifying a region with liquid-liquid phase separation (LLPS) at room temperature. Interestingly, this LLPS gives rise to micron-sized domains, whose size and stability against coalescence depend on the composition of the aqueous medium. LLPS was not expected to take place in this kind of medium since normally the presence of surfactants and/or salts destabilizes conventional LLPS structures, such as coacervates and vesicles [52,53]. Moreover, these LLPS microdomains were able to confine different active principles to a larger extent than simple micelles, including hydrophobic (poly(dimethylsiloxane) bis(3-aminopropyl)) and hydrophilic ((Z,Z)-disodium distyryl-biphenyl disulfonate) molecules. The encapsulation of active principles with different water affinities in a surfactant-rich medium is a very interesting property, generally difficult to achieve with simple coacervation, making this copolymer a promising candidate for industrial formulations. Whether the use of PEG-g-PVAc is intended for fundamental studies on self-coacervation or for applicative purposes to form microcapsules in complex formulations, a thorough understanding and control of the compositions and conditions inducing the LLPS of this copolymer is needed.

In this study, we explore the effect of several water-soluble additives on the phase behavior of PEGg-PVAc, namely kosmotropic and chaotropic compounds, as well as non-ionic, anionic, and cationic surfactants. We show that the copolymer's CPT displays a strong dependence on the chemical nature and concentration of these additives, in line with the reported CPTs of other LCST copolymers $[23,54,55]$. We then explore the effect of sodium citrate and a $C_{14-15} E_{7}$ non-ionic surfactant (N45-7) on the phase behavior of PEG-g-PVAc, defining the phase boundaries for the generation of LLPS microdomains at room temperature and monitoring their formation with fluorescence confocal microscopy. Finally, we determine the composition of the LLPS microdomains through confocal Raman microscopy. The ensemble of results defines the optimal composition and medium conditions to obtain LLPS microdomains with encapsulation potential at room temperature in surfactant-rich formulations 


\section{MATERIALS \& METHODS}

\section{Materials}

Sodium chloride ( $\mathrm{NaCl}$ ), Mw $58.4 \mathrm{~g} / \mathrm{mol}$, purity $\geq 99 \%$; Sodium bromide ( $\mathrm{NaBr}$ ), $\mathrm{Mw} 102.9 \mathrm{~g} / \mathrm{mol}$, purity 299\%; Dodecyltrimethylammonium chloride (DTAC), $\mathrm{Mw} 263.9 \mathrm{~g} / \mathrm{mol}$, purity >98\%; Dodecyltrimethylammonium bromide (DTAB), Mw $308.3 \mathrm{~g} / \mathrm{mol}$, purity $>98 \%$; Rhodamine B Isothiocyanate, mixed isomers, Mw $536.1 \mathrm{~g} / \mathrm{mol}$, were purchased from Sigma-Aldrich.

Trisodium citrate dihydrate ( $\mathrm{NaCit}$ ), Mw $294.1 \mathrm{~g} / \mathrm{mol}$, purity $>98 \%$, Jungbunzlauer; Sodium cumene sulfonate (NaCS) 40\%, Mw $222.2 \mathrm{~g} / \mathrm{mol}$, Sasol; Monopropylene glycol (MPG), Mw $76.1 \mathrm{~g} / \mathrm{mol}$, INEOS Oxide; Urea, Mw $60.1 \mathrm{~g} / \mathrm{mol}$, VWR International; Neodol 45-7 (N45-7), primary alcohol ethoxylate, C14-15 E07, Mw 519-573 g/mol, Shell Chemical; Surfonic L24-9 (L24-9), primary alcohol ethoxylate, C12-14 E09, Mw 582-610 g/mol (theoretical), Huntsman; Cetyltrimethylammonium bromide (CTAB), Mw $364.5 \mathrm{~g} / \mathrm{mol}$, purity $>99 \%$, Sigma-Aldrich; Cetyltrimethylammonium chloride (CTAC), Mw 320 $\mathrm{g} / \mathrm{mol}$, purity $96 \%$, Alfa Aesar; SOLFODAC AC-3-H (HLAS), linear alkylbenzene sulfonate, C10-13, Mw 312-360 g/mol, Tellerini s.p.a.; Tensagex EOC970B (AE3S), sodium lauryl ether sulphate $70 \%$, EO3, $\mathrm{Mw} 420 \mathrm{~g} / \mathrm{mol}$, KLK Tensachem S.A., were provided by Procter \& Gamble.

Poly(ethylene glycol)-graft-poly(vinyl acetate) (PEG-g-PVAc) from BASF was also provided by Procter \& Gamble. The copolymer has a PEG/PVAc weight ratio of $40 / 60, M n=10.3 \mathrm{kDa}, \mathrm{Mw}=18.1 \mathrm{kDa}$ (thus, with a polydispersity index (PDI) of 1.8). This copolymer has approximately one grafting point every 50 units of ethylene glycol, meaning that there are 2-3 grafted PVAc chains per PEG backbone (which is of $6 \mathrm{kDa}$ ). PEG-g-PVAc was covalently labelled with rhodamine B isothiocyanate, according to a previously described procedure [38].

All reagents were used as received without any further purification. The water used throughout this work was of MilliQ grade $\left(18.2 \mathrm{M} \Omega . \mathrm{cm}\right.$ at $\left.25^{\circ} \mathrm{C}\right)$

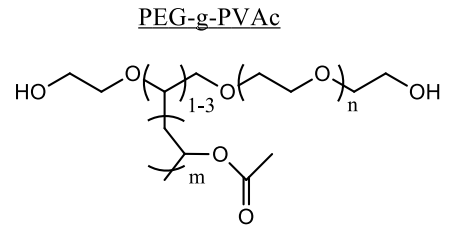

Linear alkylbenzene sulfonate (HLAS)<smiles>CCCCCCCCC(CCC)c1ccc(S(=O)(=O)[O-])cc1</smiles>

$\underline{\text { Sodium cumene sulfonate }} \underline{(\mathrm{NaCS})}$<smiles>CC(C)c1ccc(S(=O)(=O)[O-])cc1</smiles>

$\underline{\text { Sodium citrate }(\mathrm{NaCit})}$<smiles>C=C([O-])C(O)(CC(=O)[O-])CC(=O)[O-]</smiles>

\section{Cetyltrimethylammonium} chloride (CTAC)

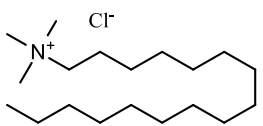

Dodecyltrimethylammonium chloride (DTAC)<smiles>CCCCCCCCCCCC[N+](C)(C)C</smiles>

Cetyltrimethylammonium bromide (CTAB)

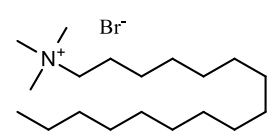

Dodecyltrimethylammonium bromide $($ DTAB)<smiles>CCCCCCCCCCC[N+](C)(C)C</smiles>

Sodium lauryl ether sulfate (AE3S)<smiles>CCCCCCCCCCCCOCCOS(=O)(=O)[O-]</smiles>

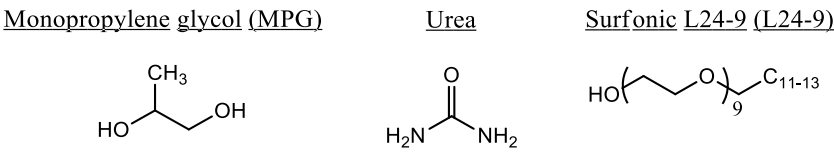

Neodol 45-7 (N45-7)

$\underline{\text { Sodium chloride }}$ $\mathrm{Na}^{+} \mathrm{Cl}^{-}$ 


\section{Samples' preparation}

PEG-g-PVAc, a wax-like solid with a melting point above room temperature, was firstly warmed at 70 ${ }^{\circ} \mathrm{C}$ for better manipulation. Then, appropriate amounts of molten PEG-g-PVAc, water, and the selected components for study were weighted in a glass vial with an analytical balance (Mettler Toledo XSR603S, accuracy of $\pm 1 \mathrm{mg}$ ). The samples were then mixed with a magnetic stirrer until a homogeneous dispersion was obtained.

PEG-g-PVAc concentration was kept at $1 \% \mathrm{w} / \mathrm{w}$, unless otherwise stated.

To map the phase behavior of PEG-g-PVAc with NaCit and N45-7 (Figure 4A), five samples were prepared in glass vials containing a fixed amount of PEG-g-PVAc $(1 \% \mathrm{w} / \mathrm{w})$ and varying amounts of non-ionic surfactant N45-7 (1, 5, 10, 15 and 20\% w/w) in water. After dissolving the copolymer and the non-ionic surfactant, the samples were titrated with sodium citrate while mixing at room temperature. All samples got cloudy after a proper amount of $\mathrm{NaCit}$ was added (Table 1 ), and optical microscopy was then used to check the presence of LLPS microdomains (Figure S5). Then, more NaCit was added to achieve a liquid-liquid bulk phase separation (Table 1).

\begin{tabular}{|c|c|c|c|c|}
\hline \multicolumn{3}{|c|}{ Initial concentrations $(\% \mathrm{w} / \mathrm{w})$} & \multirow{2}{*}{$\begin{array}{c}\text { NaCit for LLPS } \\
(\% \mathrm{w} / \mathrm{w})\end{array}$} & \multirow{2}{*}{$\begin{array}{l}\text { NaCit for bulk phase } \\
\text { separation }(\% \mathrm{w} / \mathrm{w})\end{array}$} \\
\hline PEG-g-PVAc & N45-7 & NaCit & & \\
\hline 1.0 & 1.0 & 1 & $4.8 \pm 0.2$ & $10.7 \pm 0.5$ \\
\hline 1.0 & 5.0 & / & $3.8 \pm 0.2$ & $9.9 \pm 0.5$ \\
\hline 1.0 & 10 & l & $2.4 \pm 0.1$ & $9.1 \pm 0.5$ \\
\hline 1.0 & 15 & / & $1.0 \pm 0.1$ & $8.3 \pm 0.4$ \\
\hline 1.0 & 20 & / & / & $7.4 \pm 0.4$ \\
\hline
\end{tabular}

Table 1: Initial concentrations of the five samples used to study the phase behavior of PEG-g-PVAc in presence of NaCit and N45-7 (Figure 4A), and corresponding concentrations of NaCit necessary to achieve LLPS and liquid-liquid bulk phase separation

\section{Cloud point determination}

The Cloud Point Temperature (CPT) was determined with turbidimetry using a Crystalline, from Technobis. The samples were analyzed in standardized glass vials of $8 \mathrm{~mL}$, measuring transmittance at $623 \mathrm{~nm}$ between $20^{\circ} \mathrm{C}$ and $90{ }^{\circ} \mathrm{C}$, with a heating rate of $0.5^{\circ} \mathrm{C} / \mathrm{min}$ and a cooling rate of $5{ }^{\circ} \mathrm{C} / \mathrm{min}$, while mixed with a magnetic stirrer at $800 \mathrm{rpm}$. The CPT was considered as the temperature corresponding to $50 \%$ transmittance with respect to the initial and final values. Each measurement was repeated at least three times.

To study the effect of ionic surfactants on CPT, samples were prepared by adding dropwise a $0.1 \%$ $\mathrm{w} / \mathrm{w}$ solution of ionic surfactant to the sample of $1 \% \mathrm{w} / \mathrm{w}$ PEG-g-PVA, and then mixed until homogenous. The CPT was measured after every addition. At higher concentrations, samples containing HLAS, AE3S, CTAB, or CTAC showed a marked Tyndall effect that prevented measuring properly the CPT at temperatures higher than $66^{\circ} \mathrm{C}$.

\section{Optical microscopy}

The optical pictures were acquired using an optical microscope from ZEISS, model AXIO Imager A1, with an Axiocam 305 color camera from ZEISS as well, while using dry objectives of 10x/0.30 and $40 x / 0.75$. Samples were placed between glass slides to delay water evaporation.

\section{Fluorescence Confocal Microscopy}

Fluorescence confocal microscopy imaging was carried out using a Leica TCS SP8 DMi 8 confocal microscope. Rhodamine B isothiocyanate was excited with an OPSL laser at $552 \mathrm{~nm}$, and the fluorescence emission was acquired using a hybrid SMD detector with a 572-612 nm spectral window. 
Dry objectives of 20x/0.75 and 40x/0.60 were used to image all samples, which were placed in an 8well device (Lab-Tek Chambered 1.0 Borosilicate Coverglass System, Nalge Nunc International). All samples, containing a total of $0.01 \% \mathrm{w} / \mathrm{w}$ of rhodamine labelled PEG-g-PVAc, were observed one day after preparation.

\section{Confocal Raman Microscopy}

Raman spectra were acquired with a Renishaw inVia Qontor Confocal Raman Microscope, using a 532 $\mathrm{nm}$ excitation laser ( $\mathrm{Nd}$ :YAG solid state type, $50 \mathrm{~mW}, 1800 \mathrm{l} / \mathrm{mm}$ grating, working at $50 \%$ intensity), and a Renishaw Centrus 1UTR57 detector. A dry objective 50Lx/0.5 was employed for all samples, which were previously deposited on a silicon substrate to avoid detecting Raman scattering from the background and covered with a glass slide to delay water evaporation. Samples were observed with the front-illuminated CCD camera $(256 \times 1024 \mathrm{px})$. The exposure time was set to be of $10 \mathrm{~s}$ for individual spectra acquisitions and of $1 \mathrm{~s}$ for the two-dimensional intensity maps, which we rebuilt from spectra acquired every $0,5 \mu \mathrm{m}$ along both $x$ and $y$ directions. The confocal microscope (Leica DM 2700) was crucial to peak the regions of interest for acquiring the Raman spectra of the medium, the LLPS microdomains, and the precipitated copolymer. High-confocality mode was used for all measurements.

Since N45-7 and PEG-g-PVAc share some functional groups, their Raman profiles are similar (Figure S8), with main peaks in the region between 2800 and $3100 \mathrm{~cm}^{-1}$ (corresponding to $\mathrm{C}-\mathrm{H}$ bonds' stretching). Therefore, the identification of N45-7 is challenging in an environment rich in PEG-g-PVAc. The largest difference between the two profiles is found on the shoulder of N45-7 at $2852 \mathrm{~cm}^{-1}$. On the other side, the relatively intense peak at $1730 \mathrm{~cm}^{-1}$ arises from the $\mathrm{C}=0$ vibration of the ester group of PVAc, clearly not present for N45-7. Based on these differences, we were able to distinguish the presence of PEG-g-PVAc from N45-7.

A set of reference samples for PEG-g-PVAc in water $(5,10,15,20,25 \% \mathrm{w} / \mathrm{w})$ and N45-7 in water $(3,6$, $9,12,15 \% \mathrm{w} / \mathrm{w}$ ) were measured through confocal Raman microscopy to get the calibration curves and assess the PEG-g-PVAc/water and N45-7/water mass ratios, respectively. At least five spectra were acquired for each sample, focusing the beam on different spatial spots. To build the calibration curves, three peaks from the Raman profiles of the PEG-g-PVAc solutions $\left(1730,2893\right.$, and $\left.2940 \mathrm{~cm}^{-1}\right)$ and three peaks from the N45-7 solutions $\left(2852,2887\right.$, and $\left.2922 \mathrm{~cm}^{-1}\right)$ were considered. After normalizing all Raman spectra to the peak of water (at $3420 \mathrm{~cm}^{-1}$ ), the intensities of each of the three peaks selected for PEG-g-PVAc and each of the three peaks selected for N45-7 were determined. The average intensity value of each sample, together with its standard deviation, was used to plot the calibration curves, reported in Figure S9 (for PEG-g-PVAc) and Figure S10 (for N45-7). These curves were then used to infer the PEG-g-PVAc/water and N45-7/water mass ratios of other samples. It is important to notice that the values obtained are not absolute but relative - ratios of PEG-g-PVAc/water and N457/water. 


\section{RESULTS AND DISCUSSION}

\section{Effect of additives on the CPT of PEG-g-PVAC}

The measurement of the CPTs of water solutions of PEG-g-PVAc at different concentrations $(0.25,0.5$, $1,2,3,4,6,10 \% \mathrm{w} / \mathrm{w}$, see Figure S1) revealed an LCST-type profile with a critical temperature of 51 ${ }^{\circ} \mathrm{C}$, found for $1 \% \mathrm{w} / \mathrm{w}$. This concentration, corresponding to approximately $5.5 \times 10^{-4} \mathrm{~mol} / \mathrm{L}$, was then chosen to investigate the effect of selected additives. Specifically, we addressed the effect of fourteen components, commonly used in home- and beauty-care products. These can be divided in four groups: (1) kosmotropes, (2), chaotropes, (3) non-ionic surfactants, and (4) ionic surfactants. The first group includes sodium citrate ( $\mathrm{NaCit})$, sodium chloride $(\mathrm{NaCl})$, and sodium bromide $(\mathrm{NaBr})$. The second group covers sodium cumene sulfonate (NaCS), monopropylene glycol (MPG), and urea. Then, the third group includes Neodol 45-7 (N45-7) and Surfonic L24-9 (L24-9). Finally, the fourth group includes both cationic (CTAB, CTAC, DTAB, and DTAC), and anionic (HLAS, and AE3S) surfactants (see Figure1 for chemical structures). The evolution of the CPT of $1 \% \mathrm{w} / \mathrm{w}$ PEG-g-PVAc as a function of the $\mathrm{w} / \mathrm{w} \%$ concentration of these additives is plotted in Figures 2 and 3.

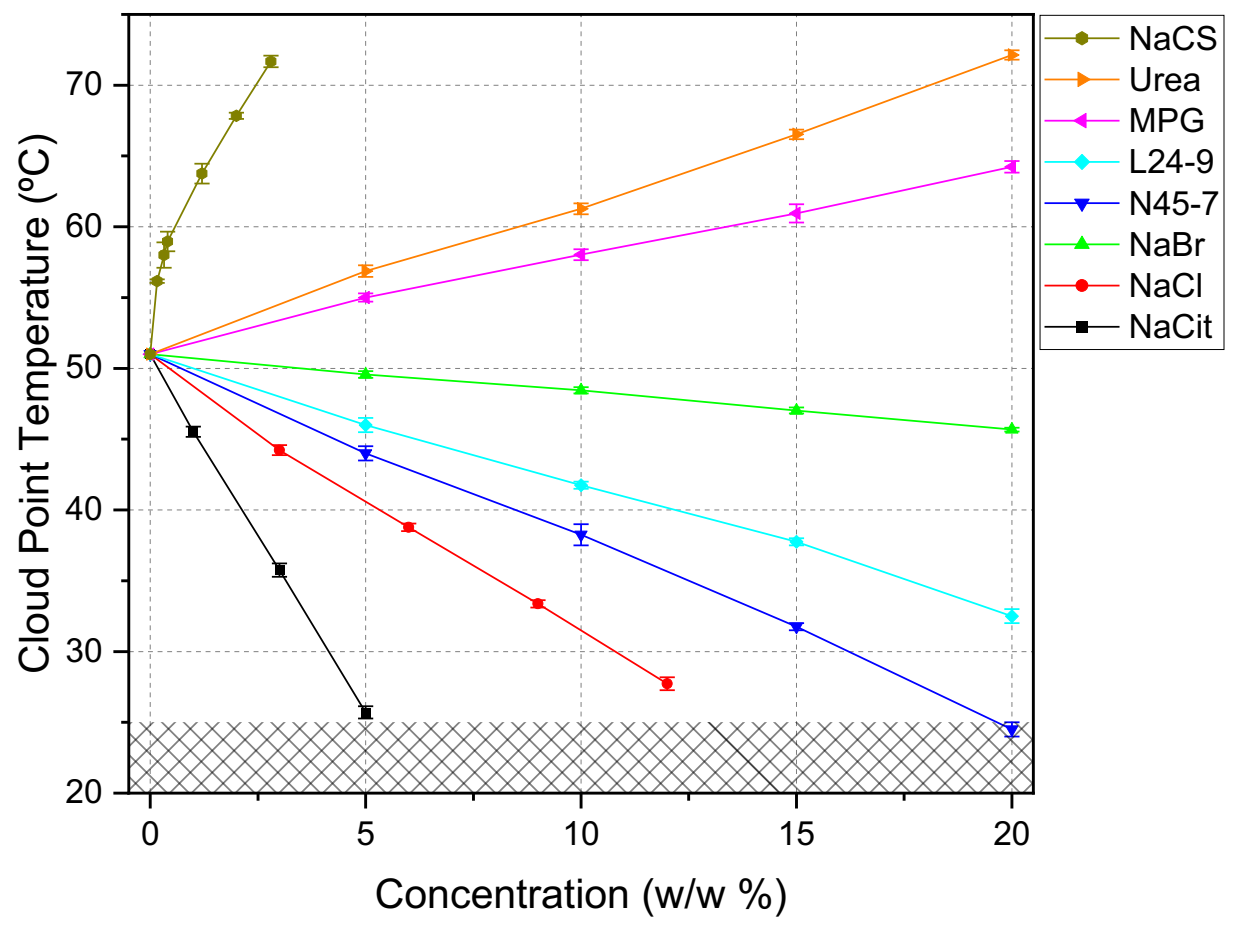

Figure 2: Effect of kosmotropes, chaotropes, and non-ionic surfactants on the CPT of $1 \% \mathrm{w} / \mathrm{w}$ PEG-g-PVAc in water measured through turbidimetry. A patterned region has been added to indicate where LLPS at room temperature would be expected. Some of the error bars are not visible because they fall within the data points drawn.

The kosmotropic additives of group 1 decrease the CPT as their concentration raises, with their effectivity following the order $\mathrm{NaCit}>\mathrm{NaCl}>\mathrm{NaBr}$. The same order is followed when their concentration is reported in $\mathrm{mol} / \mathrm{L}$ (Figure S2). It is known that both anions and cations are responsible for shifts in the CPT [55], but since the cation is shared among these salts, the different effect over the CPT is ascribed to the anions. According to the Hofmeister series and the salting-out effect [56], their different activity is related to their hydration. It is generally accepted that highly hydrated kosmotropes are "water structure-makers", increasing water surface tension and decreasing the solubility of other solutes [57]. Indeed, it has been observed that kosmotropes affect the solvency of the ethylene oxide groups of Pluronics [58]. This is in line with our findings, with $\mathrm{NaCit}$ being the most 
effective additive to reduce the CPT. Some studies have justified the decreased solvency by a direct interaction of the anions with the solute and its hydration shell $[55,59]$. Other recent studies suggest that the effect of these anions should not be defined only by their influence on the structure of water, since the same effects have been observed in non-aqueous solvents [60,61]. Therefore, a more complex interplay between water, PEG-g-PVAc, and kosmotropic agent should be considered to understand our observations on the evolution of the CPT of PEG-g-PVAc. Assuming the salt effects over the CPT follow a linear trend, a concentration of $5.1 \% \mathrm{w} / \mathrm{w}(0.17 \mathrm{~mol} / \mathrm{L})$ of $\mathrm{NaCit}$ or $13.3 \% \mathrm{w} / \mathrm{w}$ $(2.28 \mathrm{~mol} / \mathrm{L})$ of $\mathrm{NaCl}$ would be needed to phase separate $1 \% \mathrm{w} / \mathrm{w}$ of PEG-g-PVAc in water at room temperature. Remarkably, the extrapolated concentration for $\mathrm{NaCit}$ agrees with the experimental results summarized later in Figure 4.

On the other side, all the compounds from group 2 increase the CPT of PEG-g-PVAc as their concentration is raised, with their effectivity following the order of NaCs $>$ urea $>$ MPG. The same order is obeyed when measured in $\mathrm{mol} / \mathrm{L}$ (Figure S2). The different effect of these chaotropes on the CPT is due to a combination of both direct and indirect interactions with PEG-g-PVAc. At variance with kosmotropic additives, we observe a non-linear trend of the CPT for NaCS concentrations below $1 \%$ w/w (Figure 2). This non-linear behavior, previously reported for other chaotropes on PNIPAM [59], can be related to a direct binding of the anion to the copolymer, which results in a solubility increase of the latter (ion-specific effect). After saturation of the available binding sites, the effect on the CPT would solely arise from anion-water interaction, in agreement with a linear dependence. For concentrations above $1 \% \mathrm{w} / \mathrm{w}$, the chaotropic effect of NaCS can be explained by its low charge density, which translates into a reduced interaction with water and hence in a salting-in effect. Studies on the effect of NaCS over the CPT of non-ionic molecules are rather scarce, but some years ago a similar effect was observed on the CPT of an ethoxylated non-ionic surfactant (Neodol 25-9) [62].

The effect of urea over water solubility of other species has been extensively described and discussed in the literature. It is reported that its effect arises from two main mechanisms: (i) a direct one, in which the formation of hydrogen bonds between urea and water around the hydrophobic portions of the solute cause the displacement of some water molecules from the hydration shell, resulting in a solubility increase; and (ii) an indirect one, whereby urea acts as a water structure breaker, facilitating the hydration of the solute $[57,63,64]$. Although the latter has been widely accepted, several studies have proved the direct interaction of urea with the solute to be the major contribution to its increased solubility. Some have suggested that urea binds to the hydrophobic regions of the copolymer, weakening the hydrophobic interaction [65], while others have proved that urea preferentially interacts with the PEG blocks of Pluronics, enhancing its solubility [66-68]. Regarding the effect of urea over PEG-g-PVAc, the increase of its CPT is likely due to a combination of both a direct interaction of urea with the copolymer and an indirect one coming from the effect of urea over water solubility.

MPG is a short chain alcohol, whose effect on the CPT also arises from a combination of an indirect mechanism, making the medium less polar and decreasing the amphiphilicity of the copolymer, and a direct one, due to MPG's interactions with the hydrophobic blocks of the copolymer, which loosens the hydrophobic interaction between grafted chains $[57,69,70]$. 


\begin{tabular}{c|ccccc} 
Surfactant & Alkyl chain length & Ethoxy groups & Charge & CMC $\left(10^{-3} \mathrm{~mol} / \mathrm{L}\right)$ & $\mathrm{CMC}\left(10^{-3} \mathrm{w} / \mathrm{w} \%\right)$ \\
\hline N45-7 & $14-15$ & 7 & $/$ & $0.010[71]$ & 0.55 \\
L24-9 & $12-14$ & 9 & $/$ & $0.03[72]$ & 2 \\
AE3S & $\approx 12$ & 3 & - & $0.70[73]$ & 29 \\
HLAS & $\approx 12$ & $/$ & - & $1.2[74]$ & 40 \\
DTAB & $\approx 12$ & $/$ & + & $15.27[75]$ & 470.8 \\
DTAC & $\approx 12$ & $/$ & + & $23.00[75]$ & 607.0 \\
CTAB & $\approx 16$ & $/$ & + & $1.00[76]$ & 36.5 \\
CTAC & $\approx 16$ & $/$ & + & $1.30[76]$ & 41.6
\end{tabular}

Table 2: Basic characteristics of the eight surfactants used throughout this study. Their CMCs are expressed both in mol/L and $w / w \%$ for ease of comprehension

The other components that decrease the CPT are the non-ionic surfactants N45-7 and L24-9. Their basic features are summarized in Table 2. In line with the components of group 1, the addition of either N45-7 or L24-9 causes a depression of the CPT (Figure 2) since both promote copolymercopolymer over copolymer-medium interactions. This can be attributed both to the effect of these surfactants in dropping water solvency and to the formation of copolymer-surfactant complexes due to hydrophobic interactions $[77,78]$. Indeed, previous works have justified this CPT depression with the presence of copolymer-surfactant mixed micelles interconnected through polymer chain bridging [79-82], also known as the "necklace and bead" model. In regard, the depression of the CPT of PEGg-PVAc should be mainly coming from hydrophobic interactions between the non-ionic surfactant and the PVAc grafts of the copolymer. There is, however, some interaction between the non-ionic surfactant and the PEG backbone that is likely collaborating on the depression of the CPT too, as previously demonstrated [83].

The higher effectivity of N45-7 over L24-9 in decreasing the CPT of PEG-g-PVAc can be explained by its shorter ethoxy chain, resulting in a weaker interaction with water, thus a higher association with the copolymer and an enhanced stability of the copolymer-surfactant complex [84]. This lower hydrophilicity is consistent with the lower CMC and LCST of N45-7 (Table 2), the latter being approximately $24{ }^{\circ} \mathrm{C}$ lower than that of L24-9 (Figure S1). Additionally, the fact that the mixture of PEG-g-PVAc and either of these non-ionic surfactants presents CPT values below those of the two individual components (Figure S1) proves the associative copolymer-surfactant interaction.

In some copolymer-surfactant systems, a low concentration of surfactant leads to the formation of mixed micelles, causing a decrease in the CPT, while a higher concentration of surfactant promotes the formation of surfactant micelles around single hydrophobic chains of the copolymer, increasing back the CPT [84-86]. This effect causes a minimum in the trend of the CPT as the concentration of surfactant increases. However, in the concentration range selected for this work, the CPT decreases linearly with the addition of the two non-ionic surfactants (Figure 2). This behavior could be ascribed to a higher affinity between PVAc blocks than between PVAc and non-ionic surfactant. The extrapolation of their linear trend allows predicting that a concentration of $19.8 \% \mathrm{w} / \mathrm{w}(0.363 \mathrm{~mol} / \mathrm{L})$ of $\mathrm{N} 45-7$ or $28.9 \% \mathrm{w} / \mathrm{w}(0.485 \mathrm{~mol} / \mathrm{L})$ of L24-9 is needed to phase separate $1 \% \mathrm{w} / \mathrm{w}$ of PEG-g-PVAc in water at room temperature (see Figure S2 for concentrations in $\mathrm{mol} / \mathrm{L}$ ). Remarkably, the extrapolated concentration for N45-7 agrees with the experimental results summarized later in Figure 4. 

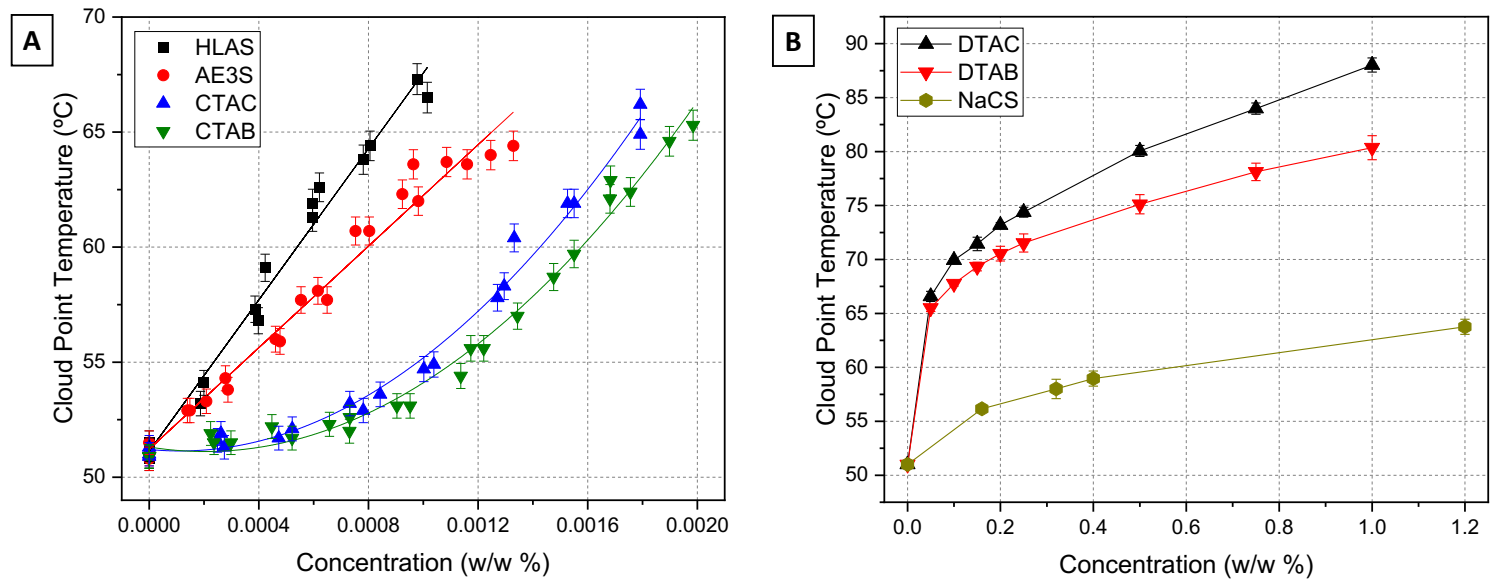

Figure 3: Effect of ionic surfactants on the CPT of 1\% w/w PEG-g-PVAc in water, measured through turbidimetry, including (A) the two anionic surfactants (HLAS and AE3S), plus the two cationic surfactants with a longer alkyl chain (CTAC and $(T A B)$, and (B) the two cationic surfactants with a shorter alkyl chain (DTAC and DTAB) plus NaCS. Some of the error bars are not visible because they fall within the data points drawn.

The last group of additives includes six ionic surfactants, consisting of four cationics (CTAB, CTAC, DTAB, and DTAC), and two anionics (HLAS and AE3S). Among them, DTAB, DTAC, HLAS, and AE3S share the same alkyl chain length (12 carbon atoms) while CTAB and CTAC have a longer alkyl chain (16 carbon atoms). Their CMCs are reported in Table 2 . Figure 3 shows that all six ionic surfactants increase the CPT of PEG-g-PVAc, and the effect is much stronger than that observed for the chaotropic additives. The same increased solubility was previously reported for a PEG-g-PVAc with a Mw $=13100$ $\mathrm{Da}$ [38], and a synergistic interaction has been recently observed at the air-water interface between an anionic surfactant (sodium dodecyl sulfate) and PEG-g-PVAc [51].

Figure $3 \mathrm{~A}$ includes the four ionic surfactants that induced the highest increase on the CPT of PEG-gPVAc. The same graph with concentrations in $\mathrm{mol} / \mathrm{L}$ can be found in the supplementary (igire S3). Their effect over the CPT is remarkable: to detect the CPT increase in a measurable temperature range, concentrations below their CMCs (Table 2) had to be used. The two anionic surfactants (HLAS and $A E 3 S$ ) were slightly more effective in increasing the CPT than the two cationic surfactants (CTAB and CTAC), even though these latter two have a longer alkyl chain. The increase of the CPT follows a linear trendline for anionic surfactants, while a lag phase followed by a rather linear trendline was observed for both CTAB and CTAC. This trendline evolution has been previously reported for other polymersurfactant systems $[87,88]$. Unfortunately, a Tyndall effect was noticed once the CPT reached levels above $66^{\circ} \mathrm{C}$, which prevented to properly study the effect of higher concentrations of these ionic surfactants over the CPT.

Figure 3B shows the effect of the other two cationic surfactants (DTAB and DTAC) over the CPT. These two ionic surfactants increased the CPT of PEG-g-PVAc to a minor degree, which allowed the comparison of their effect over the CPT with $\mathrm{NaCS}$ (see Figure S4 for concentrations in $\mathrm{mol} / \mathrm{L}$ ). Even though the alkyl chain length of these two cationic surfactants is as long as that of HLAS and AE3S, there is a noticeable difference in their effect over the CPT - while around $0.0012 \% \mathrm{w} / \mathrm{w}\left(3 \times 10^{-5} \mathrm{~mol} / \mathrm{L}\right)$ of anionic surfactant is enough to increase the CPT of PEG-g-PVAc to 65 degrees, close to $0.05 \% \mathrm{w} / \mathrm{w}$ $\left(2 \times 10^{-3} \mathrm{~mol} / \mathrm{L}\right)$ of DTAB or DTAC is needed to obtain the same increase. It can be observed that the evolutions of the CPT of PEG-g-PVAc as a function of the concentrations of DTAB, DTAC, or NaCS follow a similar trend: parabolic at low concentrations and rather linear at higher ones. No Tyndall effect was observed when using these two cationic surfactants, which permitted assessing the effect of higher concentrations. 
These results agree with the literature on the effect of ionic surfactants on the CPT of copolymers from the ethylene oxide family [23,70,89-91]. Generally, a significant increase on the CPT of watersoluble copolymers is observed after addition of either anionic or cationic surfactants. This phenomenon was justified with the formation of a copolymer-surfactant complex, which can be originated from hydrophobic interactions, following the previously mentioned "necklace and bead" model [92,93], but also from electrostatic ones, considering the charged nature of the ionic surfactants' headgroups $[51,94,95]$. This "charged" copolymer-surfactant complex, which could be considered as a polyelectrolyte [96], leads to both a better solvation of the copolymer and a higher electrostatic repulsion between complexes, thus hampering aggregation and consequently raising the CPT.

Following this explanation, the fact that the two anionic surfactants increase more effectively the CPT than any of the other four cationic surfactants suggests that the copolymer-surfactant complex of PEG-g-PVAc with anionic surfactants forms at lower concentrations and possesses a higher solubilizing effect than the complex formed with cationic surfactants, presumably due to a stronger interaction of the sulfate group than the trimethylammonium group with PEG-g-PVAc. Anionic surfactants have proved to be more effective than cationic surfactants in increasing the CPT of other systems as well $[58,97,98]$. Additionally, assuming again the formation of a copolymer-surfactant complex, a longer alkyl chain provides a higher hydrophobicity to the surfactant and reduces the repulsion factor between close polar head groups, thus strengthening the interaction between the surfactant and the copolymer [99]. This explains why a longer alkyl chain brings a more noticeable increase on the CPT among the four cationic surfactants studied in this work. The same trend has been previously reported in other polymer-surfactant systems $[23,91,97]$.

Regarding the effect of counterions, the difference between bromide and chloride shows a minimum influence on the effect of CTAB and CTAC over the CPT for the concentration range studied (clearly visible in Figure $\mathrm{S} 3$ ), in agreement with the literature $[97,100]$. However, a more noticeable difference is discernible between DTAB and DTAC, which is accentuated at higher concentrations (Figure 3B). Their different effectivity in increasing the CPT of PEG-g-PVAc can be explained by the different polarizability of bromide and chloride. It is known that chaotropic counterions bind more strongly to the micellar surface than kosmotropic counterions do, hence promoting micellar growth more effectively and reducing the CMC [101-103]. Indeed, the CMC of DTAB is lower than the one of DTAC (Table 2) because bromide interacts more favorably with the positively charged cationic surfactant than chloride does. A stronger binding of the counter-ion means a higher screening of the charged surfactant and thus a lower ionization degree, leading to a lower solubilizing effect over the copolymer, hence a lower increase on the CPT.

Even though it has not been investigated in the current work, other publications have proved that the addition of salts can counteract the strong effect of ionic surfactants over the CPT of copolymers, up to a point in which they behave like a non-ionic surfactant and decrease rather than increase the CPT $[104,105]$. 


\section{LLPS microdomains of PEG-g-PVAC}

As observed, kosmotropic salts and non-ionic surfactants decrease the CPT of PEG-g-PVAc. The most effective kosmotrope (sodium citrate) and non-ionic surfactant (Neodol 45-7) were selected to further explore PEG-g-PVAc LLPS when simultaneously present. Figure 4A presents a mapping of the compositional space of a water solution of $1 \% \mathrm{w} / \mathrm{w}$ PEG-g-PVAc. Four main scenarios occur, depending on the medium composition: homogeneous (monophasic) dispersion (1); formation of LLPS microdomains of PEG-g-PVAc with the absence (2) or presence (3) of precipitated copolymer; and liquid-liquid bulk phase separation (4). The experimental procedure used to assess the boundaries between regions of Figure 4A is explained in the materials and methods section. In short, NaCit was stepwise added to five samples, each containing $1 \% \mathrm{w} / \mathrm{w}$ PEG-g-PVAc and varying concentrations of N45-7. The blue line separating region 1 from 2 and 3 marks the appearance of a cloudy suspension, while the upper red line identifies the boundary from cloudy to biphasic.
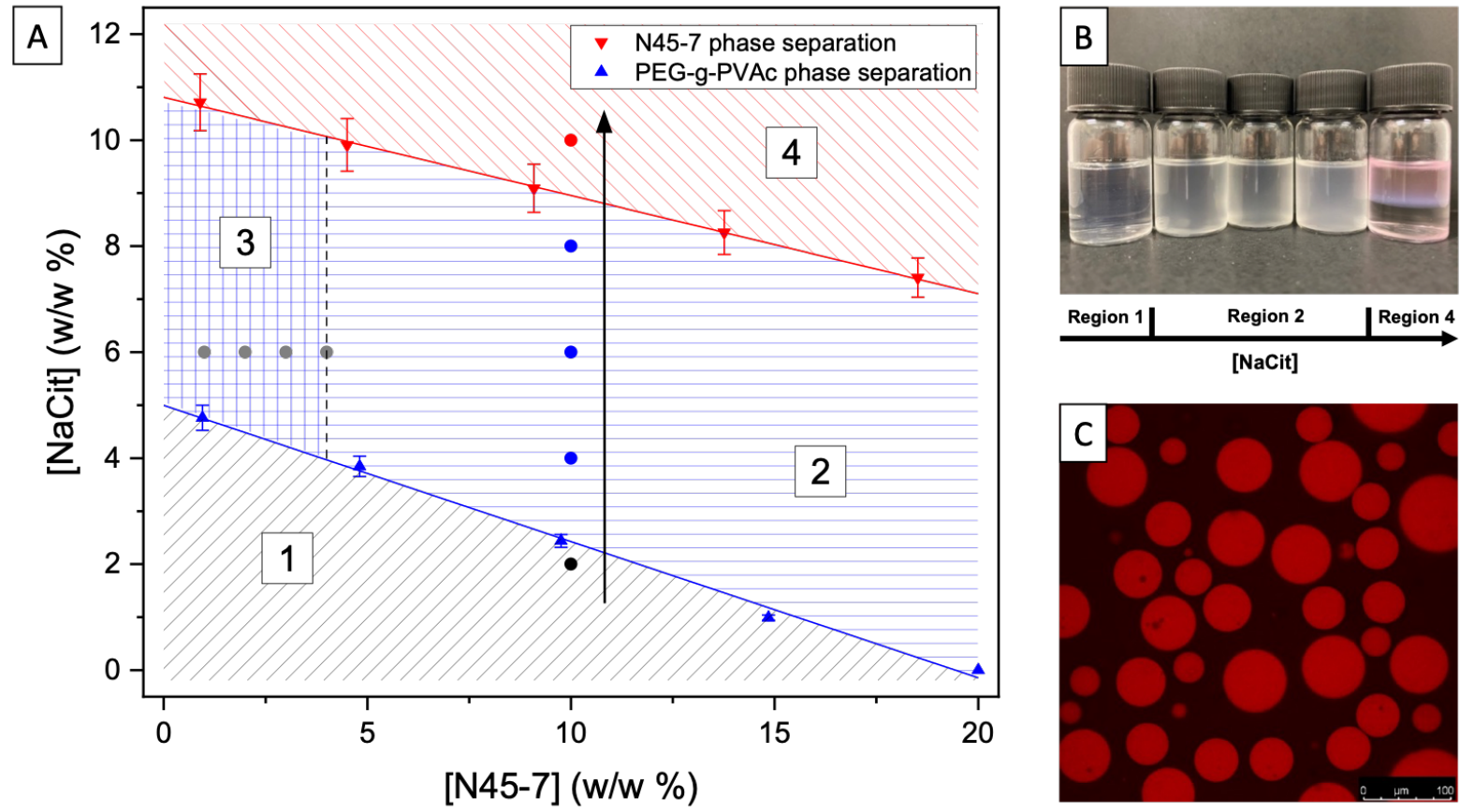

Figure 4: A water solution of 1\% w/w PEG-g-PVAc shows four different behaviors depending on the concentrations of both NaCit and N45-7 (A): in region 1, the copolymer is dissolved along the medium, showing a monophasic system; in region 2,

LLPS of the copolymer takes place giving rise to kinetically stable microdomains; in region 3, LLPS microdomains are distinguished together with copolymer precipitation; finally, in region 4, liquid-liquid bulk phase separation takes place with the copolymer being found in the top phase. In (B), five samples with constant concentrations of PEG-g-PVAc $(1 \% \mathrm{w} / \mathrm{w})$ and N45-7 (10\% w/w), but different concentrations of NaCit, are shown to illustrate the optical difference between regions 1, 2, and 4. From left to right, the first sample shows a monophasic system, the next three show a cloudy system, while the last one shows a biphasic system. In the latter sample, rhodamine-labelled PEG-g-PVAc is used to prove its exclusive presence in the top phase. In (C), fluorescence confocal microscopy is employed to observe a sample with $1 \% \mathrm{w} / \mathrm{w}$ rhodamine-labelled PEG-g-PVAc, 10\% w/w N45-7, and 5\% w/w NaCit, presenting LLPS microdomains.

Since PEG-g-PVAc is a LCST-type copolymer, a monophasic system was expected for low concentrations of $\mathrm{NaCit}$ and N45-7, where its CPT is still above room temperature, rendering the copolymer soluble in water. However, after adding a proper amount of these two components, liquidliquid phase separation takes place, caused by the depression of the copolymer's CPT. In these conditions, optical microscopy showed the presence of micron-sized globular LLPS domains (Figure $\underline{\text { S5). }}$. The difference between regions 2 and 3, both containing micron-sized LLPs domains, stems from the absence or presence of solid copolymer precipitates, respectively. The simultaneous presence of 
liquid-liquid and liquid-solid phase separation has also been detected and investigated for complex coacervates [106-108].

Interestingly, both additives lower the CPT in ternary systems (igire 2), but while the addition of N457 gives rise to LLPS microdomains, NaCit induces liquid-solid phase separation. We ascribe the different effect of these two additives to a higher degree of copolymer desolvation in the presence of $\mathrm{NaCit}$, as later indicated in Figure $5 \mathrm{~B}$. This also indicates that N45-7 is necessary to form LLPS microdomains. Moreover, if the concentrations of both PEG-g-PVAc (1\% w/w) and NaCit $(6 \% \mathrm{w} / \mathrm{w})$ are kept constant and the dispersion is titrated with N45-7, a visible decrease of the amount of solid PEGg-PVAc is observed (Table 3 , plotted as grey dots on region 3 of Figure 4A). The absence of PEG-g-PVAc precipitation is found from a concentration of $4 \% \mathrm{w} / \mathrm{w}$ N45-7.

According to the linear boundary separating region 1 from regions 2 and 3, 5\% w/w of NaCit or 19.6 $\% \mathrm{w} / \mathrm{w}$ of $\mathrm{N} 45-7$ is needed to phase separate PEG-g-PVAc. These two approximated concentrations are very close to the values obtained from the results of Figure 2 (these being $5.1 \% \mathrm{w} / \mathrm{w}$ and $19.8 \%$ $\mathrm{w} / \mathrm{w}$, respectively). The fact that this transition boundary follows a linear regression suggests that the combined effect of NaCit and N45-7 over the CPT of PEG-g-PVAc is not synergistic but rather the sum of their individual effects.

To understand the role of $\mathrm{NaCit}$ to obtain the different regions of Figure 4A, the phase behavior of five samples with constant concentrations of PEG-g-PVAc ( $1 \% \mathrm{w} / \mathrm{w})$ and $\mathrm{N} 45-7(10 \% \mathrm{w} / \mathrm{w})$, but different concentrations of $\mathrm{NaCit}$ (Table 3), was assessed. A day after sample preparation, three different scenarios could be distinguished in this set of samples (Figure 4B). The sample with $2 \% \mathrm{w} / \mathrm{w}$ of NaCit was transparent (region 1), the samples with 4,6 , and $8 \% \mathrm{w} / \mathrm{w}$ of NaCit were cloudy (region 2), while the sample with $10 \% \mathrm{w} / \mathrm{w}$ of $\mathrm{NaCit}$ was showing a liquid-liquid bulk separation (region 4). Rhodaminelabelled PEG-g-PVAc was used in the sample presenting bulk phase separation with the aim to find out its distribution. As observable, PEG-g-PVAc could only be distinguished in the top phase. This phenomenon is attributed to the kosmotropic-induced phase separation of the non-ionic surfactant, since a sample of $1 \% \mathrm{w} / \mathrm{w}$ PEG-g-PVAc without N45-7 did not present this liquid-liquid bulk phase separation at high $\mathrm{NaCit}$ concentrations $(12 \% \mathrm{w} / \mathrm{w})$, while a sample of $\mathrm{N} 45-7(10 \% \mathrm{w} / \mathrm{w})$ and $\mathrm{NaCit}$ $(10 \% \mathrm{w} / \mathrm{w})$ without PEG-g-PVAc did present this liquid-liquid bulk phase separation.

\begin{tabular}{ccc} 
[N45-7] $(\% \mathbf{w} / \mathbf{w})$ & [NaCit] $(\% \mathbf{w} / \mathbf{w})$ & Sample state \\
\hline 1 & 6 & Extended precipitation + LLPS microdomains \\
2 & 6 & Precipitation + LLPS microdomains \\
3 & 6 & Scarce precipitation + LLPS microdomains \\
4 & 6 & LLPS microdomains \\
10 & 2 & Monophasic \\
10 & 4 & LLPS microdomains \\
10 & 6 & LLPS microdomains \\
10 & 8 & LLPS microdomains \\
10 & 10 & Liquid-liquid bulk phase separation
\end{tabular}

Table 3: Composition of the two sets of samples used to determine the limit between regions 2 and 3 (minimum of N45-7), as well as to show the visual differences between regions 1, 2 and 4.

To further study the role of the kosmotropic effect on the formation of LLPS microdomains, another set of five samples with a fixed concentration of PEG-g-PVAc $(1 \% \mathrm{w} / \mathrm{w})$ and different concentrations of $\mathrm{NaCl}$ was studied (Figure S6). After adding the proper amount of N45-7, LLPS microdomains were successfully obtained in all five samples, which presented the same morphological features as the ones obtained with $\mathrm{NaCit}$. These results demonstrate that $\mathrm{NaCit}$ is not specifically needed to obtain 
LLPS microdomains, but just a kosmotrope (such as $\mathrm{NaCl}$ ) to decrease the CPT of PEG-g-PVAc and allow their formation at lower temperatures.

To demonstrate the presence of PEG-g-PVAc in these microstructures, a water solution with $1 \% \mathrm{w} / \mathrm{w}$ PEG-g-PVAc (rhodamine labelled), 5\% w/w NaCit, and 10\% w/w N45-7 was observed through fluorescence confocal microscopy (Figure $4 \mathrm{C}$ ). This instrument allowed the distinction of well-defined globular structures, with a typical diameter around $50 \mu \mathrm{m}$. The strong red fluorescence of the LLPS microdomains indicates the enrichment of PEG-g-PVAc. Moreover, the copolymer appears to be homogeneously distributed within these microdomains, not following a core-shell structure. Due to the resolution of this technique, particles with a size below approximately $500 \mathrm{~nm}$ cannot be distinguished. However, considering previous studies [36], the presence of sub-micron structures made of PEG-g-PVAc and/or N45-7 is likely. It is relevant to add that these LLPS microdomains are kinetically stable, and thus sedimentation and coalescence will take place over time. For this specific sample, the microphase separation was stable for at least five days at room temperature. The kinetic stability of this system can be extended by either increasing the concentration of surfactant or adding a structuring agent, which consequently increases the sample's viscosity and slows down the diffusion of these microstructures. Additional fluorescence confocal microscopy pictures of these LLPS microdomains can be found in the supplementary information (Figure S7). 


\section{Confocal Raman microscopy analysis of PEG-g-PVAC LLPS microdomains}

Fluorescence confocal microscopy (Figure 4B and Figure S7) suggests that the LLPS microdomains contain PEG-g-PVAc. However, this technique cannot determine the distribution of N45-7 nor assess the presence of water in the microstructure. Therefore, we selected a representable LLPS sample ( $1 \%$ $\mathrm{w} / \mathrm{w}$ PEG-g-PVAc, $5 \% \mathrm{w} / \mathrm{w} \mathrm{NaCit}$, and $10 \% \mathrm{w} / \mathrm{w} \mathrm{N} 45-7)$ for further investigation through confocal Raman microscopy. Reference Raman spectra of pure PEG-g-PVAc and N45-7 can be found in the supplementary information (Figure S8). As expected, the Raman spectrum obtained from the LLPS microdomains differs with respect to the one from the suspending medium (Figure 5A). The presence of PEG-g-PVAc (main peak at $2940 \mathrm{~cm}^{-1}, \mathrm{C}-\mathrm{H}$ stretching), water (broad peak around $3420 \mathrm{~cm}^{-1}, \mathrm{O}-\mathrm{H}$ stretching), and N45-7 (little shoulder around $2852 \mathrm{~cm}^{-1}, \mathrm{C}-\mathrm{H}$ stretching) can be appreciated for LLPS microdomains, while the medium shows mainly the presence of N45-7 (with its three diagnostic peaks at 2852, 2887, and $2922 \mathrm{~cm}^{-1}$, originating from different $\mathrm{C}-\mathrm{H}$ stretching modes), and water. We cannot rule out the presence of PEG-g-PVAc dissolved in the medium, but its occurrence does not emerge in this Raman analysis. From these results, we can qualitatively conclude that the LLPS microdomains show an enrichment of PEG-g-PVAc, in line with fluorescence confocal microscopy, while N45-7 is slightly more concentrated in the medium, as the comparison of the peaks' intensities at $2852 \mathrm{~cm}^{-1}$ indicates (inset of Figure 5A).
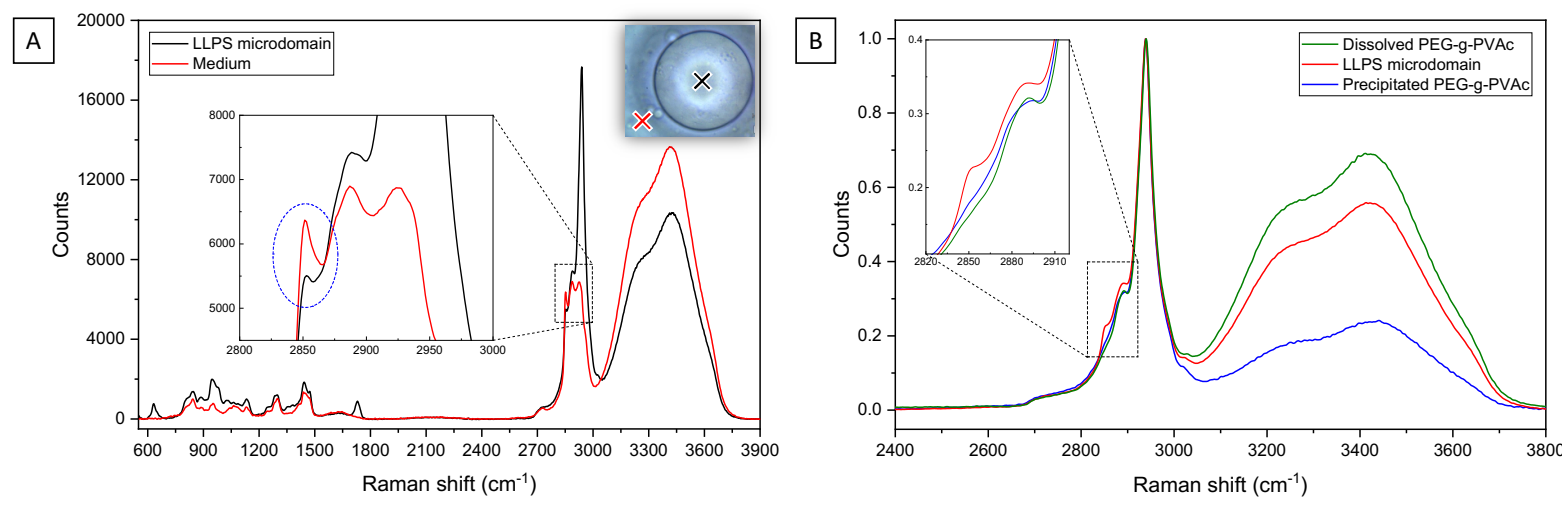

Figure 5: The sample made of $1 \% \mathrm{w} / \mathrm{w}$ PEG-g-PVAc, $5 \% \mathrm{w} / \mathrm{w}$ NaCit, and $10 \% \mathrm{w} / \mathrm{w} \mathrm{N} 45-7$ presented LLPS microdomains. In (A), Raman profiles of a LLPS microdomain (black) and the medium (red). The inset shows the three N45-7 diagnostic peaks, highlighting the different intensity of the $2852 \mathrm{~cm}^{-1}$ one. An optical microscope picture of a LLPS microdomain is enclosed at the top right corner of this figure, marking the spots from where the two Raman spectra were acquired. In (B), the Raman profiles of different samples containing PEG-g-PVAc are normalized at $2940 \mathrm{~cm}^{-1}$ for comparison. The Raman profile of the precipitated copolymer from a sample of $1 \% \mathrm{w} / \mathrm{w}$ PEG-g-PVAc and $6 \% \mathrm{w} / \mathrm{w}$ NaCit appears in blue, the averaged profile of several LLPS microdomains is in red, while the one of $25 \% \mathrm{w} / \mathrm{w}$ PEG-g-PVAc in water is in green.

Since all the diagnostic peaks from N45-7 are in a spectral range where also PEG-g-PVAc presents Raman scattering, the accurate detection of the non-ionic surfactant in the microdomains is challenging. To address this issue, at least fifty Raman spectra from the LLPS microdomains, each normalized with respect to the main peak of PEG-g-PVAc $\left(2940 \mathrm{~cm}^{-1}\right)$, were averaged and compared to those of two reference samples: (i) 25\% w/w PEG-g-PVAc in water, and (ii) precipitated copolymer from $1 \% \mathrm{w} / \mathrm{w}$ PEG-g-PVAc and $6 \% \mathrm{w} / \mathrm{w}$ NaCit (Figure $5 \mathrm{~B}$ ). The spectrum of pure PEG-g-PVAc can be found in Figure $\mathrm{S} 8$ for comparison. The Raman profiles of these three samples presented some obvious differences in the region of the $\mathrm{O}-\mathrm{H}$ stretching of water (between 3000 and $3700 \mathrm{~cm}^{-1}$ ) and $\mathrm{C}-\mathrm{H}$ stretching of alkanes (between 2800 and $3100 \mathrm{~cm}^{-1}$ ). Regarding the water region of the spectra, the LLPS microdomains still have a high content of water, as highlighted by a comparison with the reference samples (PEG-g-PVAc dissolved in water and precipitated). This result is expected and in line with other reports pointing that coacervates have a high content of water $[109,110]$. Moreover, the decreased water content of the precipitated copolymer sample suggests that this liquid-solid phase 
separation is ascribed to a higher desolvating effect of $\mathrm{NaCit}$ than N45-7, as previously commented for the results of Figure 4. For the alkane region, the spectra from $25 \% \mathrm{w} / \mathrm{w}$ PEG-g-PVAc in water and that from precipitated PEG-g-PVAc are very similar. However, when comparing the averaged spectrum obtained from the LLPS microdomains to these two other profiles, we can distinguish a shoulder around $2852 \mathrm{~cm}^{-1}$ and an increased intensity around $2887 \mathrm{~cm}^{-1}$ (inset of Figure 5B). These differences are attributed to the presence of N45-7 inside the LLPS microdomains.

To better highlight the partition of N45-7 in the microdomains, a two-dimensional Raman intensity map was performed on the sample containing $1 \% \mathrm{w} / \mathrm{w}$ PEG-g-PVAc, $5 \% \mathrm{w} / \mathrm{w} \mathrm{NaCit}$, and $10 \% \mathrm{w} / \mathrm{w}$ N457 (Figure 6A). The collection of Raman spectra was processed to obtain three different intensity distributions: one from the most intense Raman peak of PEG-g-PVAc $\left(2940 \mathrm{~cm}^{-1}\right)$, one from the ester peak of PEG-g-PVAc $\left(1730 \mathrm{~cm}^{-1}\right)$, and one from the N45-7 peak $\left(2852 \mathrm{~cm}^{-1}\right)$ - which appear in red, blue, and green, respectively, in Figure 6A. The intensity measured for PEG-g-PVAc at $2940 \mathrm{~cm}^{-1}$ can include a contribution from N45-7, which also shows Raman scattering in this region, while the intensity at $1730 \mathrm{~cm}^{-1}$ is selective for PEG-g-PVAc. These intensity maps show clearly that the microdomains are rich in PEG-g-PVAc, in line with the fluorescence confocal microscopy images. On the other side, the intensity map at $2852 \mathrm{~cm}^{-1}$ (Figure 6A, green profile) indicates a higher concentration of N45-7 in the medium than in the LLPS microdomains, which agrees with the spectral profiles of Figure 5A. It must also be noted that both PEG-g-PVAc and N45-7 have Raman scattering at $2852 \mathrm{~cm}^{-1}$. Therefore, the (green) intensity map of this emission wavelength originates both from the copolymer and the nonionic surfactant. However, as seen from the distributions at 2940 and $1730 \mathrm{~cm}^{-1}$, (in red and blue, respectively), PEG-g-PVAc is mainly concentrated inside the LLPS microdomains. Therefore, we can assume that the intensity contribution in the medium is mainly from N45-7.

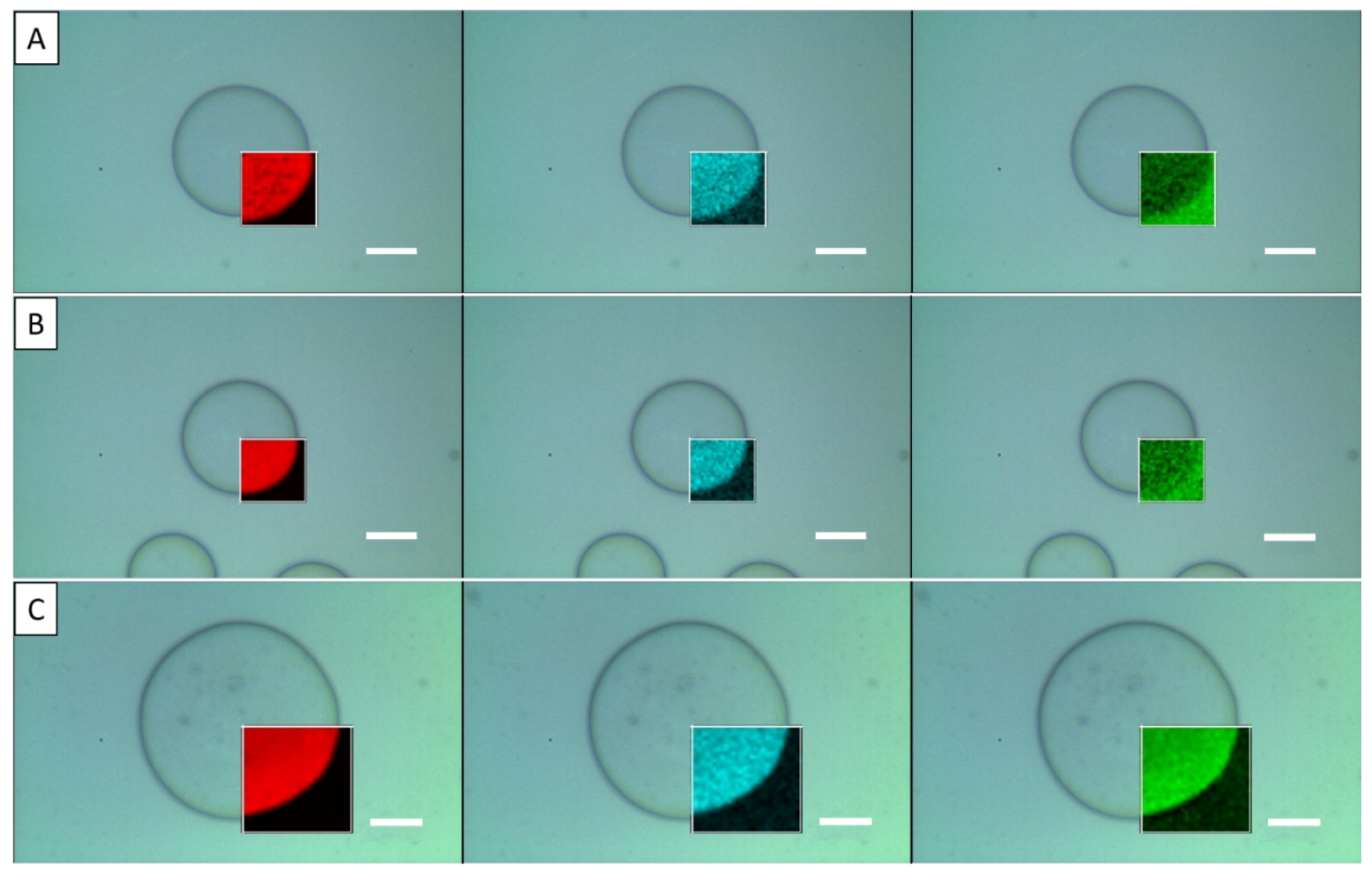

Figure 6: Two-dimensional intensity maps obtained from the Raman intensity of $1 \% \mathrm{w} / \mathrm{w}$ PEG-g-PVAc, 5\% $w / w$ NaCit, and $10 \%(A), 7 \%(B)$, or $4 \%(C) w / w ~ N 45-7$. The red distribution represents the Raman intensity measured at $2940 \mathrm{~cm}^{-1}$, the blue represents the intensity at $1730 \mathrm{~cm}^{-1}$, and the green represents the intensity at $2852 \mathrm{~cm}^{-1}$.

The scale bar is equivalent to $20 \mu \mathrm{m}$ in all pictures. 
To better understand the effect of N45-7, we assessed two additional samples with $7 \% \mathrm{w} / \mathrm{w}$ and $4 \%$ w/w N45-7 (Figures 6B and 6C, respectively), keeping unvaried the concentrations of PEG-g-PVAc (1\% $\mathrm{w} / \mathrm{w})$ and $\mathrm{NaCit}(5 \% \mathrm{w} / \mathrm{w})$. These intensity maps confirm again the localization of PEG-g-PVAc in the LLPS microdomains. Interestingly, the main difference between this series of samples is the intensity map at $2852 \mathrm{~cm}^{-1}$ (green), presenting either a higher, similar, or lower intensity in the LLPS microdomains with respect to the medium for the samples with $4 \%, 7 \%$ and $10 \% \mathrm{w} / \mathrm{w}$ of $\mathrm{N} 45-7$, respectively. Clearly, the more N45-7 in the formulation, the higher its presence in the medium rather than in the LLPS microdomains. This trend suggests that the N45-7/PEG-g-PVAc mass ratio might not vary much between LLPS microdomains, regardless of the bulk N45-7 concentration.

To address this point, the averages of at least fifty Raman spectra from the LLPS microdomains of the $7 \%$ and $4 \% \mathrm{w} / \mathrm{w}$ N45-7 samples, normalized with respect to the main peak of PEG-g-PVAc $\left(2940 \mathrm{~cm}^{-1}\right)$, were compared with: (i) the averaged spectrum of the LLPS microdomains in the $10 \% \mathrm{w} / \mathrm{w} \mathrm{N} 45-7$ sample, and (ii) a reference spectrum of $25 \% \mathrm{w} / \mathrm{w}$ PEG-g-PVAc in water (Figure 7 ). This comparison highlights once more the presence of the shoulder around $2852 \mathrm{~cm}^{-1}$ in the microdomains, not present for $25 \% \mathrm{w} / \mathrm{w}$ PEG-g-PVAc in water (inset of Figure 7). However, the intensity increase provided by N457 is small for all three concentrations, hence the amount of N45-7 in the LLPS microdomains must be low. Bearing this in mind, it can be assumed that the Raman intensity obtained from the inside of the LLPS microdomains in the intensity maps measured at $2852 \mathrm{~cm}^{-1}$ is mainly coming from PEG-g-PVAc, not from the N45-7 (shown with a green color in Figure 6). Furthermore, considering the closeness between the Raman profiles of these three samples in the alkane region (between 2800 and $3100 \mathrm{~cm}^{-}$ ${ }^{1}$ ), we can conclude that the N45-7/PEG-g-PVAc mass ratio inside the LLPS microdomains is very similar for the three different N45-7 concentrations. Therefore, an increased N45-7 concentration does not result in its noticeable enrichment inside the LLPS microdomains. The same behavior was described for coacervates made of $\mathrm{C}_{12} \mathrm{E}_{10}$ non-ionic surfactant, where its concentration in the coacervate phase changed very little with increasing its initial concentration [111]. In addition, a semi-quantitative assessment of the N45-7/PEG-g-PVAc mass ratio in the LLPS microdomains yielded an approximate value between 0.1 and 0.2 (Figure S9).

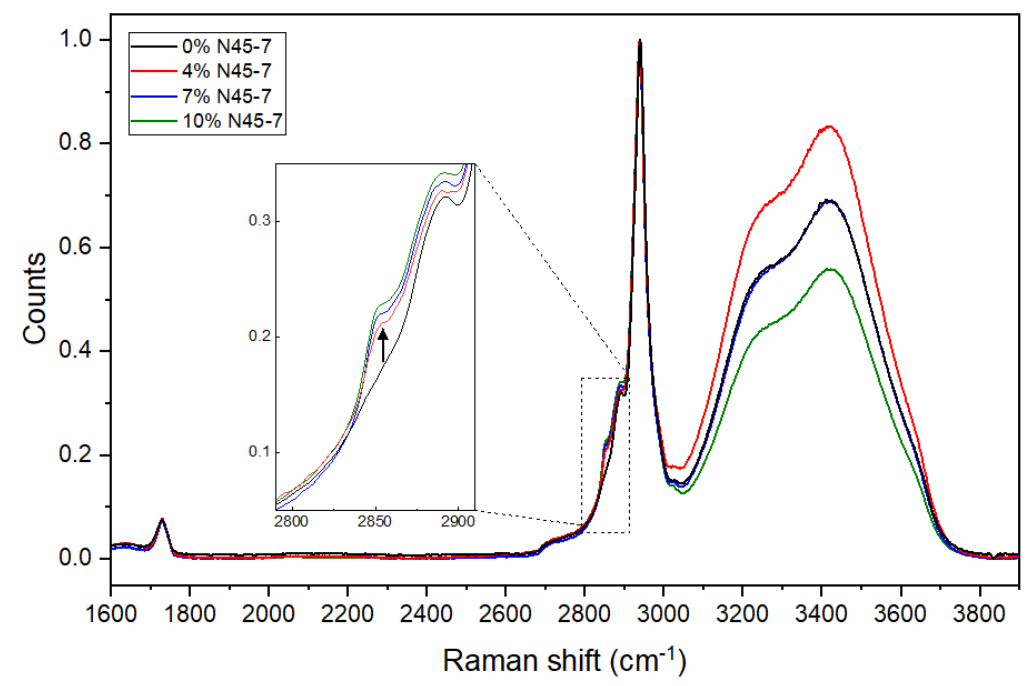

Figure 7: Averaged Raman profiles of the LLPS microdomains from three different samples consisting of $1 \% w / w$ PEG- $g$ PVAc, $5 \%$ w/w NaCit, and 4 (red), 7 (blue), or $10 \%$ (green) $w / w$ N45-7. The reference Raman profile of $25 \% w / w$ PEG-g-PVAc in water (black) is added to notice the effect of N45-7, which is marked with a vertical black arrow in the inset.

Interestingly, the most noticeable difference between the three samples of Figure 7 is the water/PEGg-PVAc content of the microdomains, which decreases as the total concentration of N45-7 increases. 
This indicates the non-ionic surfactant to be causing a decrease of the hydration within LLPS microdomains. A similar effect was reported for $\mathrm{NaCl}$ on the water content of simple coacervates [109]. This relates to the cloud point trend since, as previously shown in Figure 2, the CPT decreases as the concentration of non-ionic surfactant increases. The lower the copolymer's CPT, the lower its affinity for water, which correlates with these Raman profiles showing a lower amount of water inside the PEG-g-PVAC LLPS microdomains as more N45-7 is in solution. This suggests that by controlling the amount of non-ionic surfactant in solution we can modulate the hydrophilicity of the LLPS microdomains and, thus, their affinity for actives with different hydrophobicity.

Finally, aiming to roughly quantify the relative amounts of PEG-g-PVAC and N45-7 in the LLPS microdomains and the medium, a set of reference samples of PEG-g-PVAc in water (Figure S10) and another set of N45-7 in water (Figure S11) were used to obtain calibration curves for the PEG-gPVAc/water and N45-7/water mass ratios, respectively. Taking advantage of their linear trend, the PEG-g-PVAc/water and N45-7/water mass ratios from the references were extrapolated to the three studied samples with different N45-7 concentrations (Table 4). The extrapolation is described in detail in the materials and methods section. Even though these calibration curves cannot be used to obtain absolute concentration values, they allow to determine that all studied LLPS microdomains have a PEG-g-PVAc/water mass ratio of at least 0.25 , which is equivalent to a sample made of $20 \% \mathrm{w} / \mathrm{w}$ PEGg-PVAc in water. This outcome agrees with the results shown in Figure 7 , in which the sample containing $7 \% \mathrm{w} / \mathrm{w} \mathrm{N} 45-7$ has an identical Raman profile with respect to $25 \% \mathrm{w} / \mathrm{w}$ PEG-g-PVAc in water, suggesting that the PEG-g-PVAc/water ratio of these two samples is very similar. Considering that all samples were prepared with an initial PEG-g-PVAc bulk concentration of $1 \% \mathrm{w} / \mathrm{w}$, the concentration of copolymer in the LLPS microdomains is at least twenty times higher. The increase of the PEG-g-PVAc/water mass ratio in the LLPS microdomains at higher N45-7 bulk concentrations is attributed to the decreased hydration, as previously commented for the results of Figure 7 . At the same time, assuming once again that there is a negligible amount of PEG-g-PVAc dissolved in the medium, the extrapolation from these calibration curves also allows to conclude that the N45-7/water mass ratio in the medium is practically the N45-7 initial bulk ratio in each of the three different samples (Table 4). The outcomes of the extrapolation are in line with previous observations: (i) the PEG-g-PVAc is highly concentrated in the LLPS microdomains, with a relative concentration over water much larger than the one set during sample preparation, and (ii) a higher N45-7 bulk concentration results in its higher presence in the medium rather than in the LLPS microdomains, as suggested by the intensity maps at $2852 \mathrm{~cm}^{-1}$ (Figure 6).

\begin{tabular}{c|cc|ccc}
\multicolumn{3}{c|}{ Initial ratios (\%) } & \multicolumn{4}{c}{ Measured ratios (\%) } \\
\cline { 2 - 6 } N45-7 & $\begin{array}{c}\text { PEG-g-PVAc } \\
\text { /water }\end{array}$ & $\begin{array}{c}\text { N45-7 } \\
\text { /water }\end{array}$ & $\begin{array}{c}\text { PEG-g-PVAc/water } \\
\text { (in LLPS) }\end{array}$ & $\begin{array}{c}\text { PEG-g-PVAc/water } \\
\text { (in medium) }\end{array}$ & $\begin{array}{c}\text { N45-7/water } \\
\text { (in medium) }\end{array}$ \\
\hline 4 & 1.1 & 4.4 & $27 \pm 1$ & N/D & $4.5 \pm 0.2$ \\
7 & 1.1 & 8.0 & $35 \pm 2$ & N/D & $7.7 \pm 0.3$ \\
10 & 1.2 & 11.9 & $43 \pm 2$ & N/D & $12.2 \pm 0.4$
\end{tabular}

Table 4: PEG-g-PVAc/water and N45-7/water mass ratios of $1 \% \mathrm{w} / \mathrm{w}$ PEG-g-PVAc, $5 \% \mathrm{w} / \mathrm{w}$ NaCit, and 4, 7, or $10 \% \mathrm{w} / \mathrm{w}$ N45-7. The initial ratios refer to the ones set during sample preparation for both PEG-g-PVAc and N45-7 over water. The measured ratios, which have been obtained from extrapolation of the calibration curves from Figures S10 and S11, consist of both N45-7 over water in the medium and PEG-g-PVAC over water in the LLPS microdomains and in the medium. 


\section{CONCLUSIONS}

In this contribution, we addressed the conditions for liquid-liquid phase separation (LLPS) of PEG-gPVAc in water, to understand and control the formation of LLPS microdomains at room temperature. We determined the effect of several additives over the cloud point temperature (CPT) of PEG-g-PVAc, showing that kosmotropes decrease the CPT ( $\mathrm{NaCit}>\mathrm{NaCl}>\mathrm{NaBr}$ ), while chaotropes increase it ( $\mathrm{NaCS}$ $>$ urea > MPG). The addition of non-ionic surfactants also reduces the CPT, with the most hydrophobic surfactant showing a higher effectivity (N45-7 > L24-9). Conversely, ionic surfactants markedly increase the CPT, with anionic surfactants being the most effective (HLAS $\approx A E 3 S>C T A C \approx C T A B>$ DTAC > DTAB). Similar trends were reported on the CPT evolution of other LCST copolymers $[54,55,57,91]$.

This set of results suggests that a combination of kosmotropes and non-ionic surfactants is optimal to promote the phase separation of PEG-g-PVAc at room temperature. In these conditions, we investigated the phase behavior of PEG-g-PVAc water solutions in presence of N45-7 and NaCit to monitor the formation of LLPS microdomains. We observed that LLPS microdomains can be easily obtained at room temperature, provided that three conditions are met: (i) the CPT of PEG-g-PVAc is decreased below room temperature, (ii) enough N45-7 (at least $4 \% \mathrm{w} / \mathrm{w}$ ) is added to avoid PEG-gPVAc precipitation, and (iii) the concentration of NaCit is kept below a threshold value to prevent liquid-liquid bulk phase separation. The easy formation of LLPS microdomains at room temperature in the presence of salts and surfactants makes PEG-g-PVAc an interesting candidate for industrial applications, even more considering its proven ability to confine and encapsulate hydrophobic active molecules, such as fragrances $[38,49]$.

Finally, we used confocal Raman microscopy to define for the first time the composition of these LLPS microdomains, finding that their main component is PEG-g-PVAc, but they also contain water and N457. Interestingly, the amount of non-ionic surfactant in the microdomains did not significantly change when increasing its bulk concentration. This offers possible benefits for industrial formulations, where it is preferred to keep some surfactant in the medium for cleaning purposes. We also observed that the hydration of the LLPS microdomains decreases as the total concentration of N45-7 increases. This suggests that the amount of non-ionic surfactant can modulate the hydrophilicity of these microdomains, and thus their affinity for actives with different water affinities.

The results here gathered expand our knowledge on self-coacervation of grafted copolymers and define the conditions in which LLPS microdomains can be obtained in a surfactant-rich medium, which can be easily implemented in the body-/house-care, cosmetic, and food fields. Our findings sum up on the emerging interest to effectively control the formation of LLPS microdomains $[112,113]$ and be used as possible in vitro models to mimic membrane-less organelles of living cells [114]. In addition, the comprehension of the formation of PEG-g-PVAC LLPS microdomains also brings directional insights on how to destabilize them, providing the first step towards a future study for the triggered release of any active molecule contained within them.

\section{ACKNOWLEDGEMENTS}

The authors acknowledge financial support from the European Union's Horizon 2020 research and innovation programme under the SAMCAPS project (grant agreement no. 814100). This work was performed in collaboration with the Procter \& Gamble Company, in Brussels, and the Consorzio Interuniversitario per lo Sviluppo dei Sistemi a Grande Interfase (CSGI), in Florence. The authors are grateful to Keo-Oudone Seymany, Constantina Sofroniou, Aleksandra Zawadka, Dr. Paolo Tempesti, and Prof. Piero Baglioni for fruitful discussions. 


\section{BIBLIOGRAPHY}

[1] X. Zhang, L. Chen, K.H. Lim, S. Gonuguntla, K.W. Lim, D. Pranantyo, W.P. Yong, W.J.T. Yam, Z. Low, W.J. Teo, H.P. Nien, Q.W. Loh, S. Soh, The Pathway to Intelligence: Using Stimuli-Responsive Materials as Building Blocks for Constructing Smart and Functional Systems, Adv. Mater. 31 (2019) 1804540. https://doi.org/10.1002/adma.201804540.

[2] O. Bertrand, J.-F. Gohy, Photo-responsive polymers: synthesis and applications, Polym. Chem. 8 (2017) 52-73. https://doi.org/10.1039/C6PY01082B.

[3] G. Kocak, C. Tuncer, V. Bütün, PH-Responsive polymers, Polym. Chem. 8 (2017) 144-176. https://doi.org/10.1039/c6py01872f.

[4] M.C. García, lonic-strength-responsive polymers for drug delivery applications, in: Stimuli Responsive Polym. Nanocarriers Drug Deliv. Appl., Elsevier, 2019: pp. 393-409. https://doi.org/10.1016/B978-0-08-101995-5.000143.

[5] M. Wei, Y. Gao, X. Li, M.J. Serpe, Stimuli-responsive polymers and their applications, Polym. Chem. 8 (2017) 127143. https://doi.org/10.1039/C6PY01585A.

[6] P. Bawa, V. Pillay, Y.E. Choonara, L.C. du Toit, Stimuli-responsive polymers and their applications in drug delivery, Biomed. Mater. 4 (2009) 022001. https://doi.org/10.1088/1748-6041/4/2/022001.

[7] A.K. Teotia, H. Sami, A. Kumar, Thermo-responsive polymers, in: Switch. Responsive Surfaces Mater. Biomed. Appl., Elsevier, 2015: pp. 3-43. https://doi.org/10.1016/B978-0-85709-713-2.00001-8.

[8] D. Roy, W.L.A. Brooks, B.S. Sumerlin, New directions in thermoresponsive polymers, Chem. Soc. Rev. 42 (2013) 7214. https://doi.org/10.1039/c3cs35499g.

[9] C. Zhao, Z. Ma, X.X. Zhu, Rational design of thermoresponsive polymers in aqueous solutions: A thermodynamics map, Prog. Polym. Sci. 90 (2019) 269-291. https://doi.org/10.1016/j.progpolymsci.2019.01.001.

[10] H. Kim, S. Lee, M. Noh, S.H. Lee, Y. Mok, G. Jin, J.-H. Seo, Y. Lee, Thermosensitivity control of polyethlyenimine by simple acylation, Polymer (Guildf). 52 (2011) 1367-1374. https://doi.org/10.1016/j.polymer.2011.01.045.

[11] P.J. Roth, F.D. Jochum, F.R. Forst, R. Zentel, P. Theato, Influence of End Groups on the Stimulus-Responsive Behavior of Poly[oligo(ethylene glycol) methacrylate] in Water, Macromolecules. 43 (2010) 4638-4645. https://doi.org/10.1021/ma1005759.

[12] E.A. Clark, J.E.G. Lipson, LCST and UCST behavior in polymer solutions and blends, Polymer (Guildf). 53 (2012) 536545. https://doi.org/10.1016/j.polymer.2011.11.045.

[13] G. Pasparakis, C. Tsitsilianis, LCST polymers: Thermoresponsive nanostructured assemblies towards bioapplications, Polymer (Guildf). 211 (2020) 123146. https://doi.org/10.1016/j.polymer.2020.123146.

[14] J. Seuring, S. Agarwal, Polymers with Upper Critical Solution Temperature in Aqueous Solution: Unexpected Properties from Known Building Blocks, ACS Macro Lett. 2 (2013) 597-600. https://doi.org/10.1021/mz400227y.

[15] Q. Zhang, C. Weber, U.S. Schubert, R. Hoogenboom, Thermoresponsive polymers with lower critical solution temperature: from fundamental aspects and measuring techniques to recommended turbidimetry conditions, Mater. Horizons. 4 (2017) 109-116. https://doi.org/10.1039/C7MH00016B.

[16] A.Z. Naqvi, Kabir-ud-Din, Clouding phenomenon in amphiphilic systems: A review of five decades, Colloids Surfaces B Biointerfaces. 165 (2018) 325-344. https://doi.org/10.1016/j.colsurfb.2018.01.060.

[17] B. Lindman, G. Karlström, Nonionic polymers and surfactants: Temperature anomalies revisited, Comptes Rendus Chim. 12 (2009) 121-128. https://doi.org/10.1016/j.crci.2008.06.017.

[18] Q. Li, A.P. Constantinou, T.K. Georgiou, A library of thermoresponsive PEG-based methacrylate homopolymers: How do the molar mass and number of ethylene glycol groups affect the cloud point?, J. Polym. Sci. 59 (2021) 230-239. https://doi.org/10.1002/pol.20200720.

[19] R.D.C. Cruz, R.J. Martins, M.J.E. de M. Cardoso, O.E. Barcia, Volumetric Study of Aqueous Solutions of Polyethylene Glycol as a Function of the Polymer Molar Mass in the Temperature Range 283.15 to $313.15 \mathrm{~K}$ and $0.1 \mathrm{MPa}$, J. Solution Chem. 38 (2009) 957-981. https://doi.org/10.1007/s10953-009-9388-1.

[20] A. Saraiva, O. Persson, A. Fredenslund, An experimental investigation of cloud-point curves for the poly(ethylene glycol)/water system at varying molecular weight distributions, Fluid Phase Equilib. 91 (1993) 291-311. https://doi.org/10.1016/0378-3812(93)85105-U.

[21] S.K. Han, B.H. Jhun, Effect of additives on the cloud point of polyethylene glycols, Arch. Pharm. Res. 7 (1984) 1-9. https://doi.org/10.1007/BF02856915.

[22] K. Knop, R. Hoogenboom, D. Fischer, U.S. Schubert, Poly(ethylene glycol) in Drug Delivery: Pros and Cons as Well as Potential Alternatives, Angew. Chemie Int. Ed. 49 (2010) 6288-6308. https://doi.org/10.1002/anie.200902672.

[23] R. Sharma, P. Bahadur, Effect of different additives on the cloud point of a polyethylene oxide-polypropylene oxide-polyethylene oxide block copolymer in aqueous solution, J. Surfactants Deterg. 5 (2002) 263-268. https://doi.org/10.1007/s11743-002-0226-9.

[24] P. Patidar, S.A. Pillai, P. Bahadur, A. Bahadur, Tuning the self-assembly of EO-PO block copolymers and quercetin solubilization in the presence of some common pharmacuetical excipients: A comparative study on a linear triblock and a starblock copolymer, J. Mol. Liq. 241 (2017) 511-519. https://doi.org/10.1016/j.molliq.2017.06.035.

[25] P. Alexandridis, T. Alan Hatton, Poly(ethylene oxide) poly(propylene oxide) poly(ethylene oxide) block copolymer surfactants in aqueous solutions and at interfaces: thermodynamics, structure, dynamics, and modeling, Colloids Surfaces A Physicochem. Eng. Asp. 96 (1995) 1-46. https://doi.org/10.1016/0927-7757(94)03028-X. 
[26] S.K. Filippov, A. Bogomolova, L. Kaberov, N. Velychkivska, L. Starovoytova, Z. Cernochova, S.E. Rogers, W.M. Lau, V. V. Khutoryanskiy, M.T. Cook, Internal Nanoparticle Structure of Temperature-Responsive Self-Assembled PNIPAM- b -PEG- b -PNIPAM Triblock Copolymers in Aqueous Solutions: NMR, SANS, and Light Scattering Studies, Langmuir. 32 (2016) 5314-5323. https://doi.org/10.1021/acs.langmuir.6b00284.

[27] F. Seidi, P. Heshmati, Synthesis of a PEG-PNIPAm thermosensitive dendritic copolymer and investigation of its selfassociation, Chinese J. Polym. Sci. 33 (2015) 192-202. https://doi.org/10.1007/s10118-015-1561-y.

[28] Y.-L. Luo, W. Yu, F. Xu, L.-L. Zhang, Novel thermo-responsive self-assembly micelles from a double brush-shaped PNIPAM- $g$-(PA- $b$-PEG- $b$-PA)- $g$-PNIPAM block copolymer with PNIPAM polymers as side chains, J. Polym. Sci. Part A Polym. Chem. 50 (2012) 2053-2067. https://doi.org/10.1002/pola.25980.

[29] C.Y. Gong, S. Shi, P.W. Dong, B. Kan, M.L. Gou, X.H. Wang, X.Y. Li, F. Luo, X. Zhao, Y.Q. Wei, Z.Y. Qian, Synthesis and characterization of PEG-PCL-PEG thermosensitive hydrogel, Int. J. Pharm. 365 (2009) 89-99. https://doi.org/10.1016/j.ijpharm.2008.08.027.

[30] B. Bogdanov, A. Vidts, A. Van Den Bulcke, R. Verbeeck, E. Schacht, Synthesis and thermal properties of poly(ethylene glycol)-poly(e-caprolactone) copolymers, Polymer (Guildf). 39 (1998) 1631-1636. https://doi.org/10.1016/S0032-3861(97)00444-8.

[31] M. Qindeel, N. Ahmed, K.U. Shah, N. Ullah, Asim.ur.Rehman, New, Environment Friendly Approach for Synthesis of Amphiphilic PCL-PEG-PCL Triblock Copolymer: An Efficient Carrier for Fabrication of Nanomicelles, J. Polym. Environ. 28 (2020) 1237-1251. https://doi.org/10.1007/s10924-020-01683-1.

[32] M. Prabaharan, J.J. Grailer, D.A. Steeber, S. Gong, Thermosensitive micelles based on folate-conjugated poly(Nvinylcaprolactam)-block-poly(ethylene glycol) for tumor-targeted drug delivery, Macromol. Biosci. 9 (2009) 744753. https://doi.org/10.1002/mabi.200800366.

[33] J. Siirilä, S. Häkkinen, H. Tenhu, The emulsion polymerization induced self-assembly of a thermoresponsive polymer poly( N -vinylcaprolactam), Polym. Chem. 10 (2019) 766-775. https://doi.org/10.1039/C8PY01421C

[34] M. Qiao, D. Chen, X. Ma, Y. Liu, Injectable biodegradable temperature-responsive PLGA-PEG-PLGA copolymers: Synthesis and effect of copolymer composition on the drug release from the copolymer-based hydrogels, Int. J. Pharm. 294 (2005) 103-112. https://doi.org/10.1016/j.ijpharm.2005.01.017.

[35] N.Y. Steinman, M. Haim-Zada, I.A. Goldstein, A.H. Goldberg, T. Haber, J.M. Berlin, A.J. Domb, Effect of PLGA block molecular weight on gelling temperature of PLGA-PEG-PLGA thermoresponsive copolymers, J. Polym. Sci. Part A Polym. Chem. 57 (2019) 35-39. https://doi.org/10.1002/pola.29275.

[36] A. Bartolini, P. Tempesti, C. Resta, D. Berti, J. Smets, Y.G. Aouad, P. Baglioni, Poly(ethylene glycol)-graft-poly(vinyl acetate) single-chain nanoparticles for the encapsulation of small molecules, Phys. Chem. Chem. Phys. 19 (2017) 4553-4559. https://doi.org/10.1039/c6cp07967a.

[37] M. Mamusa, P. Tempesti, A. Bartolini, E. Carretti, A.F. Ghobadi, J. Smets, Y.G. Aouad, P. Baglioni, Associative properties of poly(ethylene glycol)-poly(vinyl acetate) comb-like graft copolymers in water, Nanoscale. 11 (2019) 6635-6643. https://doi.org/10.1039/C8NR10453K.

[38] A. Bartolini, P. Tempesti, A.F. Ghobadi, D. Berti, J. Smets, Y.G. Aouad, P. Baglioni, Liquid-liquid phase separation of polymeric microdomains with tunable inner morphology: Mechanistic insights and applications, J. Colloid Interface Sci. 556 (2019) 74-82. https://doi.org/10.1016/j.jcis.2019.08.015.

[39] C. Feng, Y. Li, D. Yang, J. Hu, X. Zhang, X. Huang, Well-defined graft copolymers: from controlled synthesis to multipurpose applications, Chem. Soc. Rev. 40 (2011) 1282-1295. https://doi.org/10.1039/B921358A.

[40] A. Ding, J. Xu, G. Gu, G. Lu, X. Huang, PHEA-g-PMMA Well-Defined Graft Copolymer: ATRP Synthesis, SelfAssembly, and Synchronous Encapsulation of Both Hydrophobic and Hydrophilic Guest Molecules, Sci. Rep. 7 (2017) 12601. https://doi.org/10.1038/s41598-017-12710-y.

[41] Y. Li, Y. Zhang, D. Yang, Y. Li, J. Hu, C. Feng, S. Zhai, G. Lu, X. Huang, PAA- g -PPO Amphiphilic Graft Copolymer: Synthesis and Diverse Micellar Morphologies, Macromolecules. 43 (2010) 262-270. https://doi.org/10.1021/ma901526j.

[42] C. Cai, J. Lin, T. Chen, X. Tian, Aggregation Behavior of Graft Copolymer with Rigid Backbone, Langmuir. 26 (2010) 2791-2797. https://doi.org/10.1021/la902834m.

[43] X. Jiang, G. Lu, C. Feng, Y. Li, X. Huang, Poly(acrylic acid)-graft-poly(N-vinylcaprolactam): a novel pH and thermo dual-stimuli responsive system, Polym. Chem. 4 (2013) 3876. https://doi.org/10.1039/c3py00415e.

[44] B. Xu, G. Gu, C. Feng, X. Jiang, J. Hu, G. Lu, S. Zhang, X. Huang, (PAA-g-PS)-co-PPEGMEMA asymmetric polymer brushes: synthesis, self-assembly, and encapsulating capacity for both hydrophobic and hydrophilic agents, Polym. Chem. 7 (2016) 613-624. https://doi.org/10.1039/C5PY01644D.

[45] A. Perro, L. Giraud, N. Coudon, S. Shanmugathasan, V. Lapeyre, B. Goudeau, J.P. Douliez, V. Ravaine, Selfcoacervation of ampholyte polymer chains as an efficient encapsulation strategy, J. Colloid Interface Sci. 548 (2019) 275-283. https://doi.org/10.1016/j.jcis.2019.04.033.

[46] R. J. Holland, A. V. York, R. M. Ruppert, Pre-treating textiles with dspersions of graft polymers based on polyalkylene oxdesto mpart sol release properties thereto, 4,999,869, 1991.

[47] S. Gopalkrishnan, E. J. Parker, R. J. Holland, S. Patterson, Detergency boosting polymer blends asadditives for laundry formulations, 5,733,856, 1998.

[48] D. Boeck, L. Herrera Taboada, A. Kavarnou-Seiler, G. Konrad, B. Reinhard, A.L. Casado-Dominguez, F. Hulskotter, J. Lee Danziger, Cleaning Compositions with Amphiphilic Graft Polymers Based on Polyalkylene Oxides and Vinyl Esters, US8143209 B2, 2012. 
[49] M. Mamusa, C. Sofroniou, C. Resta, S. Murgia, E. Fratini, J. Smets, P. Baglioni, Tuning the Encapsulation of Simple Fragrances with an Amphiphilic Graft Copolymer, ACS Appl. Mater. Interfaces. 12 (2020) 28808-28818. https://doi.org/10.1021/acsami.0c05892.

[50] M. Mamusa, R. Mastrangelo, T. Glen, S. Murgia, G. Palazzo, J. Smets, P. Baglioni, Rational Design of Sustainable Liquid Microcapsules for Spontaneous Fragrance Encapsulation, Angew. Chemie. 133 (2021) 24042-24050. https://doi.org/10.1002/ange.202110446.

[51] A. Slastanova, R.A. Campbell, T. Snow, E. Mould, P. Li, R.J.L. Welbourn, M. Chen, E. Robles, W.H. Briscoe, Synergy, competition, and the "hanging" polymer layer: Interactions between a neutral amphiphilic 'tardigrade' comb copolymer with an anionic surfactant at the air-water interface, J. Colloid Interface Sci. 561 (2020) 181-194. https://doi.org/10.1016/j.jcis.2019.11.017.

[52] U. Kragh-Hansen, M. le Maire, J. V. Møller, The Mechanism of Detergent Solubilization of Liposomes and ProteinContaining Membranes, Biophys. J. 75 (1998) 2932-2946. https://doi.org/10.1016/S0006-3495(98)77735-5.

[53] I. Insua, A. Wilkinson, F. Fernandez-Trillo, Polyion complex (PIC) particles: Preparation and biomedical applications, Eur. Polym. J. 81 (2016) 198-215. https://doi.org/10.1016/j.eurpolymj.2016.06.003.

[54] L. Pérez-Fuentes, D. Bastos-González, J. Faraudo, C. Drummond, Effect of organic and inorganic ions on the lower critical solution transition and aggregation of PNIPAM, Soft Matter. 14 (2018) 7818-7828. https://doi.org/10.1039/c8sm01679h.

[55] S.Z. Moghaddam, E. Thormann, The Hofmeister series: Specific ion effects in aqueous polymer solutions, J. Colloid Interface Sci. 555 (2019) 615-635. https://doi.org/10.1016/j.jcis.2019.07.067.

[56] W. Kunz, J. Henle, B.W. Ninham, 'Zur Lehre von der Wirkung der Salze' (about the science of the effect of salts): Franz Hofmeister's historical papers, Curr. Opin. Colloid Interface Sci. 9 (2004) 19-37. https://doi.org/10.1016/j.cocis.2004.05.005.

[57] P. Patidar, A. Bahadur, Modulating effect of different biomolecules and other additives on cloud point and aggregation of amphiphilic linear and starblock copolymer, J. Mol. Liq. 249 (2018) 219-226. https://doi.org/10.1016/j.molliq.2017.11.019.

[58] L. Xiuli, X. Jian, H. Wanguo, S. Dejun, Effect of additives on the cloud points of two tri-block copolymers in aqueous solution, Colloids Surfaces A Physicochem. Eng. Asp. 237 (2004) 1-6. https://doi.org/10.1016/j.colsurfa.2004.02.008.

[59] Y. Zhang, S. Furyk, D.E. Bergbreiter, P.S. Cremer, Specific ion effects on the water solubility of macromolecules: PNIPAM and the Hofmeister series, J. Am. Chem. Soc. 127 (2005) 14505-14510. https://doi.org/10.1021/ja0546424.

[60] K.P. Gregory, G.B. Webber, E.J. Wanless, A.J. Page, Lewis Strength Determines Specific-lon Effects in Aqueous and Nonaqueous Solvents, J. Phys. Chem. A. 123 (2019) 6420-6429. https://doi.org/10.1021/acs.jpca.9b04004.

[61] V. Mazzini, G. Liu, V.S.J. Craig, Probing the Hofmeister series beyond water: Specific-ion effects in non-aqueous solvents, J. Chem. Phys. 148 (2018) 222805. https://doi.org/10.1063/1.5017278.

[62] R.L. Burns, Hydrotropic properties of a novel alkylnaphthalene sulfonate, J. Surfactants Deterg. 2 (1999) $483-488$. https://doi.org/10.1007/s11743-999-0096-1.

[63] S. Mahbub, The impact of electrolyte and urea on the phase separation of Triton X-100, J. Mol. Liq. 307 (2020) 112912. https://doi.org/10.1016/j.molliq.2020.112912.

[64] R.C. Da Silva, W. Loh, Effect of Additives on the Cloud Points of Aqueous Solutions of Ethylene Oxide-Propylene Oxide-Ethylene Oxide Block Copolymers, J. Colloid Interface Sci. 202 (1998) 385-390. https://doi.org/10.1006/jcis.1998.5456.

[65] R. Zangi, R. Zhou, B.J. Berne, Urea's Action on Hydrophobic Interactions, J. Am. Chem. Soc. 131 (2009) $1535-1541$. https://doi.org/10.1021/ja807887g.

[66] J.H. Ma, C. Guo, Y.L. Tang, L. Chen, P. Bahadur, H.Z. Liu, Interaction of urea with pluronic block copolymers by1H NMR spectroscopy, J. Phys. Chem. B. 111 (2007) 5155-5161. https://doi.org/10.1021/jp070887m.

[67] S.A. Pillai, C.-F. Lee, D. Ray, V.K. Aswal, M.-R. Wang, L.-J. Chen, P. Bahadur, Influence of urea on single and mixed micellar systems of Tetronics ${ }^{\circledR}$, J. Mol. Liq. 252 (2018) 9-17. https://doi.org/10.1016/j.molliq.2017.12.101.

[68] E. V Lage, S.A. Pillai, H. Pal, A. Bahadur, M. Casas, I. Sández-Macho, P. Bahadur, Urea induced changes in selfassembly and aggregate microstructures of amphiphilic star block copolymers with widely different hydrophobicity, Colloids Surfaces A Physicochem. Eng. Asp. 537 (2018) 259-267. https://doi.org/10.1016/j.colsurfa.2017.10.032.

[69] B. Bharatiya, C. Guo, J.H. Ma, P.A. Hassan, P. Bahadur, Aggregation and clouding behavior of aqueous solution of EO-PO block copolymer in presence of n-alkanols, Eur. Polym. J. 43 (2007) 1883-1891. https://doi.org/10.1016/j.eurpolymj.2007.02.010.

[70] J.L. Li, D.S. Bai, B.H. Chen, Effects of additives on the cloud points of selected nonionic linear ethoxylated alcohol surfactants, Colloids Surfaces A Physicochem. Eng. Asp. 346 (2009) 237-243. https://doi.org/10.1016/j.colsurfa.2009.06.020.

[71] C. Honda, Y. Katsumata, R. Yasutome, S. Yamazaki, S. Ishii, K. Matsuoka, K. Endo, Temperature dependence of pyrene fluorescence spectra in aqueous solutions of CnEm (C14E7, C16E7, and C16E6) nonionic surfactant micelles, J. Photochem. Photobiol. A Chem. 182 (2006) 151-157.

https://doi.org/10.1016/j.jphotochem.2006.02.003.

[72] J. Venzmer, Determination of CMCs Results from CESIO / TEGEWA Working Groups, SOFW J. 146 (2020). 
https://www.tegewa.de/en/wp-content/uploads/sites/2/2021/05/Publication-CMCs-SOFW-Journal-March2020.pdf.

[73] L.A. Sanova, A.N. Lisitsyn, Simulation of foaming ability, multiplicity, and foam stability of shampoo, Russ. J. Appl. Chem. 85 (2012) 898-906. https://doi.org/10.1134/S1070427212050110.

[74] M.. García, E. Campos, M. Dalmau, I. Ribosa, J. Sánchez-Leal, Structure-activity relationships for association of linear alkylbenzene sulfonates with activated sludge, Chemosphere. 49 (2002) 279-286. https://doi.org/10.1016/S0045-6535(02)00182-0.

[75] Y. Takata, Y. Shimatsu, T. Nagahashi, T. Miyayama, A. Hyono, H. Ohshima, Effect of lonic Surfactants on Ion Vibration Current and Colloid Vibration Current, J. Oleo Sci. 59 (2010) 401-406. https://doi.org/10.5650/jos.59.401.

[76] J. Mata, D. Varade, P. Bahadur, Aggregation behavior of quaternary salt based cationic surfactants, Thermochim. Acta. 428 (2005) 147-155. https://doi.org/10.1016/j.tca.2004.11.009.

[77] J. Appell, G. Porte, M. Rawiso, Interactions between nonionic surfactant micelles introduced by a telechelic polymer. A small angle neutron scattering study, Langmuir. 14 (1998) 4409-4414. https://doi.org/10.1021/la9712395.

[78] K.-W. Zhang, G. Karlström, B. Lindman, Phase behaviour of systems of a non-ionic surfactant and a non-ionic polymer in aqueous solution, Colloids and Surfaces. 67 (1992) 147-155. https://doi.org/10.1016/01666622(92)80294-C.

[79] G. Zhao, C.C. Khin, S.B. Chen, B.-H. Chen, Nonionic Surfactant and Temperature Effects on the Viscosity of Hydrophobically Modified Hydroxyethyl Cellulose Solutions, J. Phys. Chem. B. 109 (2005) 14198-14204. https://doi.org/10.1021/jp051955c.

[80] N. Rehman, H. Ullah, S. Alam, A.K. Jan, S.W. Khan, M. Tariq, Surface and thermodynamic study of micellization of non ionic surfactant/diblock copolymer system as revealed by surface tension and conductivity, J. Mater. Environ. Sci. 8 (2017) 1161-1167.

[81] D. Bai, C.C. Khin, S.B. Chen, C.-C. Tsai, B.-H. Chen, Interaction between a Nonionic Surfactant and a Hydrophobically Modified 2-Hydroxyethyl Cellulose, J. Phys. Chem. B. 109 (2005) 4909-4916. https://doi.org/10.1021/jp045538w.

[82] A. Hashidzume, T. Ohara, Y. Morishima, Coacervation of Hydrophobically Modified Polyanions by Association with Nonionic Surfactants in Water, Langmuir. 18 (2002) 9211-9218. https://doi.org/10.1021/la020493g.

[83] E. Feitosa, W. Brown, P. Hansson, Interactions between the non-ionic surfactant C12E5 and poly(ethylene oxide) studied using dynamic light scattering and fluorescence quenching, Macromolecules. 29 (1996) 2169-2178. https://doi.org/10.1021/ma950516g.

[84] E. Alami, M. Almgren, W. Brown, Interaction of Hydrophobically End-Capped Poly(ethylene oxide) with Nonionic Surfactants in Aqueous Solution. Fluorescence and Light Scattering Studies, Macromolecules. 29 (1996) 50265035. https://doi.org/10.1021/ma9518161.

[85] P. Hansson, B. Lindman, Surfactant-polymer interactions, Curr. Opin. Colloid Interface Sci. 1 (1996) 604-613. https://doi.org/10.1016/s1359-0294(96)80098-7.

[86] R. Tanaka, J. Meadows, P.A. Williams, G.O. Phillips, Interaction of hydrophobically modified hydroxyethyl cellulose with various added surfactants, Macromolecules. 25 (1992) 1304-1310. https://doi.org/10.1021/ma00030a016.

[87] Q. Qiu, P. Somasundaran, B.A. Pethica, Hydrophobic complexation of poly(vinyl caprolactam) with sodium dodecyl sulfate and dodecyltrimethylammonium bromide in solution, Langmuir. 18 (2002) 3482-3486.

https://doi.org/10.1021/la011702k.

[88] T.F. Tadros, The interaction of cetyltrimethylammonium bromide and sodium dodecylbenzene sulfonate with polyvinyl alcohol. adsorption of the polymer-surfactant complexes on silica, J. Colloid Interface Sci. 46 (1974) 528 540. https://doi.org/10.1016/0021-9797(74)90064-2.

[89] S. Aktar, M. Saha, S. Mahbub, M.A. Halim, M.A. Rub, M.A. Hoque, D.M.S. Islam, D. Kumar, Y.G. Alghamdi, A.M. Asiri, Influence of polyethylene glycol on the aggregation/clouding phenomena of cationic and non-ionic surfactants in attendance of electrolytes ( $\mathrm{NaCl} \& \mathrm{Na} 2 \mathrm{SO} 4)$ : An experimental and theoretical analysis, J. Mol. Liq. 306 (2020). https://doi.org/10.1016/j.molliq.2020.112880.

[90] J.P. Mata, P.R. Majhi, M. Yamashita, A. Khanal, K. Nakashima, P. Bahadur, Aggregation behavior of pluronic-L64 in presence of sodium dodecyl sulphate in water, J. Dispers. Sci. Technol. 29 (2008) 1248-1256. https://doi.org/10.1080/01932690701857152.

[91] T. Patel, P. Bahadur, J. Mata, The clouding behaviour of PEO-PPO based triblock copolymers in aqueous ionic surfactant solutions: A new approach for cloud point measurements, J. Colloid Interface Sci. 345 (2010) 346-350. https://doi.org/10.1016/j.jcis.2010.01.079.

[92] S. Chatterjee, R. Prajapati, A. Bhattacharya, T.K. Mukherjee, Microscopic evidence of "necklace and bead"-like morphology of polymer-surfactant complexes: A comparative study on poly(vinylpyrrolidone)- sodium dodecyl sulfate and poly(diallyldimethylammonium chloride)-sodium dodecyl sulfate systems, Langmuir. 30 (2014) 98599865. https://doi.org/10.1021/la5022615.

[93] A. Bonincontro, P. Michiotti, C. La Mesa, Structure and Dynamics of Polymer-Surfactant Complexes: Dielectric Relaxation Studies, J. Phys. Chem. B. 107 (2003) 14164-14170. https://doi.org/10.1021/jp035326j.

[94] D.J. Cooke, J.A.K. Blondel, J. Lu, R.K. Thomas, Y. Wang, B. Han, H. Yan, J. Penfold, Interaction between Poly(ethylene oxide) and Monovalent Dodecyl Sulfates Studied by Neutron Reflection, Langmuir. 14 (1998) 1990- 
1995. https://doi.org/10.1021/la971129y.

[95] M. Cao, M. Hai, Investigation on the Interaction between Sodium Dodecyl Sulfate and Polyethylene Glycol by Electron Spin Resonance, Ultraviolet Spectrum, and Viscosity, J. Chem. Eng. Data. 51 (2006) 1576-1581. https://doi.org/10.1021/je060067n.

[96] K. Pandya, K. Lad, P. Bahadur, Effect of Additives on the Clouding Behavior of an Ethylene Oxide-Propylene Oxide Block Copolymer in Aqueous Solution, J. Macromol. Sci. Part A. 30 (1993) 1-18. https://doi.org/10.1080/10601329308009387.

[97] N. Sardar, M.S. Ali, M. Kamil, Kabir-ud-Din, Phase Behavior of Nonionic Polymer Hydroxypropylmethyl Cellulose: Effect of Gemini and Single-Chain Surfactants on the Energetics at the Cloud Point, J. Chem. Eng. Data. 55 (2010) 4990-4994. https://doi.org/10.1021/je100572d.

[98] E.J. Kim, D.O. Shah, Effect of surfactants on the cloud point of amphiphilic drug solutions, Colloids Surfaces A Physicochem. Eng. Asp. 227 (2003) 105-111. https://doi.org/10.1016/S0927-7757(03)00415-1.

[99] M.S. Bakshi, S. Sachar, K. Singh, A. Shaheen, Mixed micelle behavior of Pluronic L64 and Triton X-100 with conventional and dimeric cationic surfactants, J. Colloid Interface Sci. 286 (2005) 369-377. https://doi.org/10.1016/j.jcis.2004.12.044.

[100] B. Vyas, S.A. Pillai, S. Tiwari, P. Bahadur, Effects of head group and counter-ion variation in cationic surfactants on the microstructures of EO-PO block copolymer micelles, Colloid Interface Sci. Commun. 33 (2019) 100216. https://doi.org/10.1016/j.colcom.2019.100216.

[101] S. Manet, Y. Karpichev, D. Bassani, R. Kiagus-Ahmad, R. Oda, Counteranion Effect on Micellization of Cationic Gemini Surfactants 14-2-14: Hofmeister and Other Counterions, Langmuir. 26 (2010) 10645-10656. https://doi.org/10.1021/la1008768.

[102] T. Nakashima, T. Fujiwara, Effects of Surfactant Counter-lons and Added Salts on Reverse Micelle Formation of Cetyltrimethylammonium Surfactant Studied by Using ( $5,10,15,20$-Tetraphenylporphyrinato ) zinc ( II ) as a Probe, Anal. Sci. 17 (2001) 1241-1244.

[103] S. Berr, R.R.M. Jones, J.S. Johnson, Effect of counterion on the size and charge of alkyltrimethylammonium halide micelles as a function of chain length and concentration as determined by small-angle neutron scattering, J. Phys. Chem. 96 (1992) 5611-5614. https://doi.org/10.1021/j100192a075.

[104] J. Mata, Hydrodynamic and Clouding Behavior of Triton X-100+SDS Mixed Micellar Systems in the Presence of Sodium Chloride, J. Dispers. Sci. Technol. 27 (2006) 49-54. https://doi.org/10.1081/DIS-200066708.

[105] A. Carlsson, G. Karlstroem, B. Lindman, Synergistic surfactant-electrolyte effect in polymer solutions, Langmuir. 2 (1986) 536-537. https://doi.org/10.1021/la00070a027.

[106] F. Comert, A.J. Malanowski, F. Azarikia, P.L. Dubin, Coacervation and precipitation in polysaccharide-protein systems, Soft Matter. 12 (2016) 4154-4161. https://doi.org/10.1039/C6SM00044D.

[107] F. Comert, D. Nguyen, M. Rushanan, P. Milas, A.Y. Xu, P.L. Dubin, Precipitate-Coacervate Transformation in Polyelectrolyte-Mixed Micelle Systems, J. Phys. Chem. B. 121 (2017) 4466-4473. https://doi.org/10.1021/acs.jpcb.6b12895.

[108] F. Comert, P.L. Dubin, Liquid-liquid and liquid-solid phase separation in protein-polyelectrolyte systems, Adv. Colloid Interface Sci. 239 (2017) 213-217. https://doi.org/10.1016/j.cis.2016.08.005.

[109] F.M. Menger, B.M. Sykes, Anatomy of a Coacervate, Langmuir. 14 (1998) 4131-4137. https://doi.org/10.1021/la980208m.

[110] E. Spruijt, A.H. Westphal, J.W. Borst, M.A. Cohen Stuart, J. Van Der Gucht, Binodal compositions of polyelectrolyte complexes, Macromolecules. 43 (2010) 6476-6484. https://doi.org/10.1021/ma101031t.

[111] Y. Huang, W. Qin, X. Huo, Y. Dai, Surfactant distribution in the clouding of C12E10, Colloids Surfaces A Physicochem. Eng. Asp. 276 (2006) 228-231. https://doi.org/10.1016/j.colsurfa.2005.11.053.

[112] J.L. Bai, D. Liu, R. Wang, Self-assembly of Amphiphilic Diblock Copolymers Induced by Liquid-Liquid Phase Separation, Chinese J. Polym. Sci. (English Ed. 39 (2021) 1217-1224. https://doi.org/10.1007/s10118-021-2563-6.

[113] X. Wang, P. Zhang, L. Tian, Spatiotemporal organization of coacervate microdroplets, Curr. Opin. Colloid Interface Sci. 52 (2021) 101420. https://doi.org/10.1016/j.cocis.2021.101420.

[114] N.A. Yewdall, A.A.M. André, T. Lu, E. Spruijt, Coacervates as models of membraneless organelles, Curr. Opin. Colloid Interface Sci. 52 (2021) 101416. https://doi.org/10.1016/j.cocis.2020.101416. 